\title{
Photoinduced electron transport in dye-containing titania gel film
}

Hiromasa Nishikiori,* Rudi Agus Setiawan, Kento Miyamoto, Gagat Sukmono, Yohei Uesugi, Katsuya Teshima, Tsuneo Fujii

Department of Environmental Science and Technology, Faculty of Engineering, Shinshu University, 4-17-1 Wakasato, Nagano 380-8553, Japan

\section{Abstract}

Amorphous dye-containing titania gel films were prepared on ITO electrodes coated with a crystalline titania foundation from titanium alkoxide sols containing a dye at room temperature. Photoinduced electron transport in the amorphous titania gel film was investigated by spectroscopic and photovoltaic measurements. Influences of the structure and morphology of the multilayered film on the photoelectron transport and electrically conductive properties were discussed. The photocurrent was observed from only the layer contacting the crystalline titania foundation. The electron transport from the amorphous upper layers was limited. Steam treatment of the electrodes improved the electron transport due to crystallization of the amorphous titania to anatase accompanied by enhancement of its electrical conductivity. The efficiency of the dye-sensitized electron transport in the steam-treated titania film was close to that of the anatase film prepared by heating at $773 \mathrm{~K}$. The dye-containing titania layers functioned as efficient sensitizers. 
Keywords: Dye-containing titania; Sol-gel method; Photoinduced electron transport;

Photoelectric conversion

*Corresponding author: Hiromasa Nishikiori

Tel.: +81-26-269-5536

Fax: +81-26-269-5550

E-mail address: nishiki@shinshu-u.ac.jp 


\section{Introduction}

Organic-inorganic hybrid nanocomposites are promising materials for improving optical and electronic devices and for creating their new functions utilizing quantum effects of organic molecules or nanosized inorganic compounds. The advancement of such devices requires basic elucidation of the physicochemical properties of such nanocomposites. The photochemistry field significantly focuses on the electron transfer in photo-functional materials consisting of such nanocomposites, such as the working electrodes of dye-sensitized solar cells [1-3]. Many scientists have investigated the electron donor-acceptor interaction between the dye molecules and the titania in the dye-titania systems used for the dye-sensitized solar cells [4-15]. Electron transport is an important process in order to understand the fundamental properties of electronic devices and further develop their functions and performances.

The dye-doped amorphous gel films, as we call them, are prepared without heating from a titanium alkoxide sol containing the dye molecules by the sol-gel method [16-20]. This method allows the dye molecules to be dispersed into the network of the titanium alkoxide polymers at a molecular level. Organic dye molecules tend to be separately encapsulated into the pores of the polymer network without any aggregation as the reaction proceeds [19]. We postulated that the aged gel consists of amorphous, nanosized, and particle-like units and 
that the dye molecules exist in the nanopores of the gel, which should be called dye-containing titania.

We have investigated the photoelectric conversion properties of the dye-containing titania gel, which is different from the conventional dye-adsorbed titania [16-20]. The characteristics of the dye-containing titania system are a high dispersion of the dye and a high contact area between the dye and titania. The titania gel has a semiconductor-like quasi-conduction band structure with a low density of states [16-18]. Why the photoelectric conversion can occur in such systems is an objective of our studies. Furthermore, the effect of the hydrothermal treatment on their photoelectric conversion properties has also been investigated because it is an effective method for crystallizing the amorphous phase and improving the photoelectric conversion performance $[16,17]$. The crystallization of amorphous titania to anatase was reported to be achieved by hydrothermal treatment at a low temperature because water molecules catalyzed the rearrangement of the $\mathrm{TiO}_{6}$ octahedra [21]. However, in many cases, the crystallinity of such titania systems is quite low compared to the general titania prepared by sufficient heating. The dye molecules interacting with the titania prevented its crystallization during steam treatment due to their high dispersion in the titania. Therefore, the electrical conductivity of the steam-treated titania should be much lower. 
Our previous studies indicated that the hydrothermal treatment of a dye-containing amorphous titania film remarkably improved the photoelectric conversion efficiency due not only to its crystallization but also to the dye-titanium complex formation $[19,20]$. The electron transport process in the titania conduction band is important for the photoelectric conversion. This process depends on the state density of the conduction band of the titania and the electron density in its conduction band. In this study, the spectroscopic and photoelectric measurements of the amorphous dye-containing titania gel and steam-treated nanocrystalline titania films were conducted in order to clarify the photoinduced electron transport in the titania. We report the influence of the structure of the multilayered film and the morphology of such nanocomposites on the photoelectron transport and electrically conductive properties.

\section{Experimental}

\subsection{Materials}

Titanium tetraisopropoxide, ethanol, fluorescein, eosin $\mathrm{Y}$, hydrochloric acid, nitric acid, diethylene glycol, iodine, and lithium iodide (Wako Pure Chemicals, S or reagent grade) were used without further purification. Water was ion-exchanged and distilled. Glass plates coated with the ITO transparent electrode (AGC Fabritech) were soaked in hydrochloric acid $\left(1.0 \mathrm{~mol} \mathrm{dm}^{-3}\right)$ for $2 \mathrm{~h}$ and then rinsed with water. The electrolyte used for the electrical 
measurements consisted of a diethylene glycol solution of iodine $\left(5.0 \times 10^{-2} \mathrm{~mol} \mathrm{dm}^{-3}\right)$ and lithium iodide $\left(0.50 \mathrm{~mol} \mathrm{dm}^{-3}\right)$.

\subsection{Preparation of electrodes}

The sol-gel reaction system was prepared by mixing $5.0 \mathrm{~cm}^{3}$ of titanium tetraisopropoxide, $25.0 \mathrm{~cm}^{3}$ of ethanol, $0.21 \mathrm{~cm}^{3}$ of water, and $0.21 \mathrm{~cm}^{3}$ of concentrated nitric acid as the catalyst for the sol-gel reaction and was labeled SG-0. Fluorescein (FC) and eosin Y (EY) were individually dissolved into the SG-0 in which their concentrations were $1.0 \times 10^{-2} \mathrm{~mol} \mathrm{dm}^{-3}$, and these systems were labeled SG-FC and SG-EY, respectively. The dip-coated thin films were prepared from the systems in which the sol-gel reaction occurred over 1 day to prepare the electrodes.

The structures of the working electrodes used in this study are shown in Scheme 1. In order to prepare the electrode samples coated with the crystalline titania, the glass plates with the ITO transparent electrode were dip-coated in the dye-free system (SG-0) and then heated at $773 \mathrm{~K}$ for $30 \mathrm{~min}$. These electrodes were labeled E-0.

The E-0 was dip-coated in SG-FC and SG-EY $n$ times $(n=1-7)$ in order to prepare the n-layered dye-containing films and they were labeled WE-FCn and WE-EYn, respectively.

The SG-FC1 and SG-EY1 were dip-coated in SG-0 $n$ times $(n=1-6)$ in order to prepare the $n$-layerd dye-free films on the dye-containing films and they were labeled WE-FC1-Tn and WE-EY1-Tn, respectively. 
The E-0 was dip-coated in SG-0 $n$ times $(n=1-7)$ and then dip-coated in SG-FC and SG-EY 3 times, respectively, in order to prepare the 3-layered dye-containing films on the $n$-layerd dye-free films. These electrodes were labeled WE-Tn-FC3 and WE-Tn-EY3, respectively.

Furthermore, the working electrodes, WE-FC3-EY3 and WE-EY3-FC3, were prepared by dip-coating in SG-FC or SG-EY 3 times and then in SG-EY or SG-FC 3 times on the E-0.

The steam-treatment effects on the UV-visible absorption and photocurrent spectra of the electrode samples were investigated. Water was heated at $383 \mathrm{~K}$ and the electrode samples were exposed to the steam for 20 min. The pressure of the steam was about $140 \mathrm{kPa}$.

The E-0 was dip-coated in SG-0 $n$ times $(n=3,5$, and 7), heated at $773 \mathrm{~K}$ for $30 \mathrm{~min}$, and then dip-coated in SG-FC 3 times in order to prepare the 3-layered dye-containing films on the $n$-layered crystalline titania film. These electrodes were steam-treated for $20 \mathrm{~min}$ and labeled steam-treated WE-Ten-FC3.

\subsection{Measurements}

The electrodes samples were cut using a diamond cutter and fixed on the sample holder keeping their surface or cross section up. The surface morphology of the electrode samples was observed and their layer thickness was estimated from their cross section using a field emission scanning electron microscope (Hitachi S-4100). The UV-visible absorption spectra of the prepared electrode samples were observed using a spectrophotometer 
(Shimadzu UV-3150). The crystalline phase of the film samples was determined by $\mathrm{CuK} \alpha$ radiation using an X-ray diffractometer (Rigaku RINT-2200V). The flakes of the film samples were pressed in the $\mathrm{KBr}$ pellets and their IR spectra were obtained using an FTIR spectrophotometer (Shimadzu IRPrestige-21). The XPS spectra were observed by AlK $\alpha$ radiation using an X-ray photoelectron spectrophotometer (ULVAC PHI 5600). The DC electrical resistance of the films was measured using a digital multimeter (ADCMT 7461A).

The iodine-based electrolyte was allowed to soak into the space between the electrode sample and the counter Pt electrode. Monochromatic light (330-600 nm) obtained from a fluorescence spectrophotometer (Shimazdu RF-5300) equipped with a 150-W Xe short arc lamp (Ushio UXL-155) was irradiated onto the electrodes for the spectroscopic measurements. During the light irradiation, the short circuit currents of the electrodes were measured by an electrometer (Keithley model 617). The current values of the electrodes, the untreated and steam-treated WE-FC3, were obtained as a function of light irradiation time in order to examine their durability. The $I-V$ curves of the electrodes were measured by a potentiostat (Hokuto Denko HSV-100) during irradiation by the visible light at a wavelength longer than $400 \mathrm{~nm}$ emitted by the 150-W Xe short arc lamp using a sharp cut filter. The intensity at each wavelength of the light source was obtained using a power meter (Molectron PM500A) in order to estimate the quantum efficiency of the photocurrent from the excited dye in the electrode samples. The light intensity was confirmed to correlate with the results of the 
potassium ferrioxalate actinometry. The visible absorbance of the present electrode samples was lower than 1.0 which was sufficient to measure the number of absorbed photons in order to calculate the quantum efficiency.

\section{Results and discussion}

3.1. Visible absorption and photocurrent properties of the untreated WE-FCn and WE-EYn

The thickness of the dye-containing layer of WE-FCn and WE-EYn was ca. $70 \mathrm{~nm}$ per layer. Fig. 1 shows the visible absorption and photocurrent spectra of WE-FCn. The peak of the absorption and photocurrent spectra of the WE-FCn was located at $480 \mathrm{~nm}$. This result indicates that the main fluorescein species was the neutral or anion form (at 450-480 nm) [22]. The absorbance increased with an increase in the number of dye-containing layers. The photocurrent spectra have a shape similar to the absorption spectra of the electrode. This correspondence indicates that the current electron was produced by a process consisting of the light absorption by fluorescein and electron transfer from its excited states to the titania gel. This electron was transported to the ITO film through the crystalline titania foundation layer. The photocurrent value increased with an increase in the number of dye-containing layers up to the fifth layer and then decreased with more layers. In the case of more than 5 layers, the dye-containing layers prevented the electric conduction. There is a possibility that some of 
the upper dye-containing layers caused back electron transfer to the electrolyte because the electron cannot be transported to the crystalline titania layer due to low electrical conductivity of the dye-containing layers. Fig. 2 shows the $I-V$ curves of the WE-FCn. The short-circuit photocurrent increased and then decreased with the number of dye-containing layers as shown in the photocurrent spectra, although the open-circuit voltage gradually increased and became similar to the values previously observed in the amorphous titania gel $[19,20]$. The open-circuit voltage was due to the semiconducting characteristics of the titania gel.

(Figs. 1 and 2)

Fig. 3 shows the visible absorption and photocurrent spectra of WE-EYn. The peak of the absorption and photocurrent spectra of the WE-EYn was at $530 \mathrm{~nm}$. The main eosin $\mathrm{Y}$ species was the dianion form due to its $\mathrm{p} K_{\mathrm{a}}$ value [23]. The absorbance and photocurrent values increased with an increase in the number of dye-containing layers. Fig. 4 shows the $I-V$ curves of the WE-EYn. Also, the short-circuit photocurrent and open-circuit voltage gradually increased. The photocurrent values for the EY-containing electrodes were lower than those for the FC-containing electrodes containing the same number of layers. The photocurrent for the EY-containing electrodes did not attain the maximum for the FC-containing electrode or its saturation. 
The photoelectric conversion efficiency depended on the dye-titanium interaction and the electron transfer process $[19,20]$. The proton dissociation constants, $\mathrm{p} K_{\mathrm{a}}$ values, of the carboxyl groups of fluorescein and eosin $\mathrm{Y}$ are 4.45 and 3.75, respectively [23]. The carboxylate oxygen of fluorescein is more nucleophilic than that of eosin $\mathrm{Y}$ and interacts more easily with the titanium species [20]. Additionally, the quinone-like carbonyl group of the xanthene ring of fluorescein also more easily interacted with the titanium species because the $\mathrm{p} K_{\mathrm{a}}$ values of the hydroxyl group of the xanthene ring are 6.80 for fluorescein and 2.81 for eosin $\mathrm{Y}[20,23]$. Therefore, the efficiency of the electron injection for the FC-containing electrodes was higher than that of the EY-containing ones.

(Figs. 3and 4)

\subsection{Visible absorption and photocurrent properties of the untreated WE-FC1-Tn and}

\section{WE-EY1-Tn}

Fig. 5 shows the visible absorption and photocurrent spectra of the WE-FC1-Tn. The absorption spectrum slightly changed with the number of dye-free layers due to no change in the dye amount. However, the photocurrent value increased with an increase in the number of dye-free layers even though the dye amount was constant. These values were clearly higher than those for the WE-FCn electrodes (Fig. 1b). The $I-V$ curves of WE-FC1-Tn as shown in Fig. 6 indicate that the short-circuit photocurrent and open-circuit voltage also gradually increased with an increase in the number of dye-free layers. 
(Figs. 5and 6)

Figs. 7 and 8 show the visible absorption and photocurrent spectra and the $I-V$ curves of the WE-EY1-Tn, respectively. The results of the absorption and photovoltaic measurements were essentially the same as those of the FC-containing electrodes, WE-FC1-Tn. The values for the EY-containing electrodes were lower than those for the FC-containing electrodes due to the weaker dye-titanium interaction. These values were clearly higher than those for the WE-EYn electrodes (Fig. 3b).

(Figs. 7and 8)

These results indicated that the photoconductive electrons were only from the layer contacting the titania foundation. The upper layers functioned as blocking layers for the back electron transfer to the electrolyte. Even in the dye-containing electrodes, the upper layers also functioned only as blocking layers because the electrons in the upper dye-containing layers cannot be transported to the crystalline titania layer due to their low electrical conductivity. The layered dye-free films more effectively improved the photocurrent than the dye-containing films because some of the dye-containing layers caused the back electron transfer. The dye-free titania was apparently more conductive than the dye-containing titania.

\subsection{Photocurrent properties of the untreated WE-Tn-FC3 and WE-Tn-EY3}


Fig. 9 shows the photocurrent spectra of WE-Tn-FC3 and WE-Tn-EY3. The photocurrent values were significantly decreased by inserting even one layer of the dye-free titania under the dye-containing titania layers. This result indicates that the electron transport occurred from the layer contacting the titania foundation but only slightly from the upper layers, i.e., the photoelectrons produced in the upper layers were not efficiently transported in the amorphous titania layers. The amorphous titania layer exhibited a very low electrical conductivity. The result in this experiment supports that shown in Sections 3.2 and 3.3, i.e., the upper layers functioned as blocking layers for the back electron transfer to the electrolyte.

(Fig. 9)

\subsection{Structural change in the titania by steam treatment}

The untreated titania gel film was confirmed to be structureless in morphology and amorphous based on SEM observation. Fig. 10 shows the SEM images of the titania film steam-treated for $20 \mathrm{~min}$ and the crystalline titania film prepared by heating at $773 \mathrm{~K}$ for 30 min. The steam-treated film consisted of 10-20 nm particles as previously reported [16]. The particles should be denser than those of the amorphous titania gel. The heated film consisted of almost the same-sized particles, which were densely packed. The steam-treated film exhibited a more porous morphology.

(Fig. 10) 
The XRD patterns of the untreated and steam-treated titania films and the crystalline titania film are shown in Fig. 11. No peak is found in the XRD pattern of the untreated amorphous gel film. Peaks at around $25^{\circ}$ and $38^{\circ}$ were observed in the steam-treated film, indicating that an anatase type crystal structure was produced in the film. The sharper peaks at $25.3^{\circ}, 37.8^{\circ}$, and $48.1^{\circ}$ were observed in the crystalline titania film. The size of the crystallites of these films was estimated from their full-width at half-maximum of the $25^{\circ}$ peak using Sherrer's equation. The crystallite size for the steam-treated film was ca. $5 \mathrm{~nm}$ and was much smaller than that of the crystalline titania, ca. $18 \mathrm{~nm}$. This is different from the particle size estimated from the SEM image because the hydrophilic surface of the particles and their agglomeration promote the formation of larger secondary particles.

Fig.12 shows the FTIR spectra of the untreated and steam-treated titania gel and the crystalline titania. The Ti-O stretching band located at around $800-400 \mathrm{~cm}^{-1}$ is seen in all the samples. It was reported that bands at around $800-700 \mathrm{~cm}^{-1}$ and around $650-400 \mathrm{~cm}^{-1}$ are assigned to tetrahedral and octahedral Ti-O, respectively [24-27]. The octahedral structure of anatase titania was enhanced by heating some titania samples [25,27]. The bands located at around $800-700 \mathrm{~cm}^{-1}$ are assigned to a tetrahedral $\mathrm{Ti}-\mathrm{O}$ as shown by a shoulder on the broad band in the spectrum of the untreated and steam-treated samples. The band was clearly located at a lower wavenumber side in the crystalline titania as 
previously reported $[28,29]$. These IR spectra indicated that the unit cell structure in the titania gel was slightly changed by the steam treatment but was significantly changed by heating.

A broad band at around $3300 \mathrm{~cm}^{-1}$ is assigned to the $\mathrm{O}-\mathrm{H}$ stretching of the $\mathrm{TiOH}$ group and adsorbed $\mathrm{H}_{2} \mathrm{O}[25,26,29,30]$. A band at $1620 \mathrm{~cm}^{-1}$ is assigned to the $\mathrm{O}-\mathrm{H}$ bending of the adsorbed $\mathrm{H}_{2} \mathrm{O}[29,30]$. These $\mathrm{O}-\mathrm{H}$ bands were observed in the untreated and steam-treated titania gel but were not observed in the crystalline titania. The $\mathrm{O}-\mathrm{H}$ stretching band for the steam-treated titania was broader and the peak was seen at a wavenumber somewhat higher than that for the untreated sample. This indicates that a larger amount of the water molecules was physically adsorbed on the steam-treated sample [26].

(Fig. 12)

Fig. 13 show the XPS spectra relating to the binding energy of the Ti $2 p$ and O 1 s electrons for the untreated and steam-treated titania gel and the crystalline titania. The Ti $2 p$ spectra of the untreated titania sample exhibited peaks at 464.7 and $459.2 \mathrm{eV}$ The peaks were slightly shifted to the lower energy side by steam treatment and were almost the same as that for the crystalline titania, 464.5 and $458.7 \mathrm{eV}$, assigned to the typical $\mathrm{Ti}^{4+}[31,32]$. This indicates that the charge density of $\mathrm{Ti}^{4+}$ was slightly changed during the transition from the amorphous gel to the anatase. 
The $\mathrm{O} 1 \mathrm{~s}$ peaks were observed at $530.1 \mathrm{eV}$ in the untreated sample, and these were almost the same as those of the steam-treated and crystalline samples. The spectrum for the untreated sample is broader than the others due to a large amount of hydroxyl groups exhibiting the peak at around 531.0-531.5 eV [28,30,32], as observed in its FTIR spectrum in Fig. 12. The spectrum for the steam-treated sample also has a shoulder at around $532 \mathrm{eV}$ assigned to the adsorbed water molecules $[28,30]$.

Consequently, the steam treatment caused crystallization and densification of the titania gel, which increased the density of states in the titania and improved its electrical conductivity. The untreated dye-containing titania gel is suggested to consist of amorphous, nanosized, and particle-like units and the dye molecules should individually exist in the nanopores of the gel. After steam treatment, an increase in the overall crystallinity of titania nanoparticles should result in formation of the specific crystalline nanoparticles and an increase in their surface quality to adsorb the dye molecules [18]. The spectroscopic measurements indicated that the dye molecules were highly dispersed on the titania surface without any aggregation.

(Fig. 13)

\subsection{Influence of steam treatment on the photocurrent properties of the dye-containing titania electrodes}

Fig. 14 shows the absorption and photocurrent spectra of the untreated dye-containing titania electrodes, WE-FC3, WE-EY3, WE-FC3-EY3, and WE-EY3-FC3. The absorption 
spectra of WE-FC3-EY3 and WE-EY3-FC3 were almost the same because they were the sum of the FC3 and EY3 layers. The photocurrent spectra of WE-FC3-EY3 and WE-EY3-FC3 indicated the enhanced spectra of WE-FC3 and WE-EY3, respectively. The upper layers enhanced only the photocurrent from the dye-containing layer contacting the titania foundation. This result corresponds to those of WE-FCn and WE-EYn, or WE-FC1-Tn and WE-EY1-Tn; i.e., the upper layers functioned as blocking layers for the back electron transfer to the electrolyte. The dyes in the upper layers had only a very limited effect on the photocurrent.

(Fig. 14)

Fig. 15 shows the absorption and photocurrent spectra of the steam-treated electrodes for these dye-containing titania electrodes. The steam treatment significantly enhanced the photocurrent of the electrodes due to the crystallization of the amorphous titania to anatase and the complex formation between the dye and titania even though it decreased the absorbance due to the dye desorption [16-20]. The photocurrent spectrum of WE-FC3-EY3 increased for both the FC and EY peaks due to the blocking effect for the back electron transfer by the EY-containing layers and allowing photoelectron injection from the EY-containing layers. The photocurrent spectrum of WE-EY3-FC3 indicated a significant enhancement in the photocurrent from FC. These results indicated that the steam treatment improved the electron transport from the upper layers, i.e., the electron became transported 
from the upper layers to the ITO film through the titania foundation layer. This main reason for this should be the crystallization of the amorphous titania accompanied by improvement in its electrical conductivity.

The photocurrent values for the electrodes, the untreated and steam-treated WE-FC3, were obtained as a function of light irradiation time and are shown in the supporting information (Fig. S1). The values decreased with time due to oxidative degradation of the dye molecules. The degradation rate for the steam-treated electrode was a little faster than that for the untreated one. In our previous study, the steam treatment of the dye-containing titania gel induced an increase in the rate of electron injection from the dye to the titania [18]. The dye degradation occurred because the rate of dye reduction by the electrolyte was slower than that of the dye oxidation. For durability, optimization of the electrolyte composition and the titania porous structure is required to improve the efficiency of the electron transfer between the electrolyte and dye.

(Figs. 15 and 16)

Fig. 16 shows the photocurrent spectra of the steam-treated dye-containing titania electrodes, WE-FC3 and WE-Tn-FC3. The photocurrent values were significantly decreased by inserting even one layer of the amorphous dye-free titania under the dye-containing titania layers as shown in Fig. 9. Only a limited photocurrent from the upper layers was observed in the untreated electrodes. The photocurrent values decreased 
with an increase in the number of dye-free titania layers. However, the steam treatment improved the photocurrent even in the electrode having 7 layers of the dye-free titania under the dye-containing layers.

\subsection{Electrical conductivity of the dye-containing titania electrodes}

The electrical resistance of the untreated and steam-treated titania films and the crystalline titania film was compared. The resistivity values for these films were $6.8 \times 10^{5}, 8.1 \times 10^{4}$, and $3.1 \times 10^{4} \Omega \mathrm{cm}$, respectively. The resistivity of the crystalline titania film was close to that of the titania prepared by heating at $773 \mathrm{~K}$ by the sol-gel method in the previous study [34]. In addition to this, the resistivity of the titania prepared at below $673 \mathrm{~K}$ was higher by one order than that prepared at $773 \mathrm{~K}$. The resistivity of the steam-treated film was of the same order as that of the crystalline titania film.

Fig. 17 shows the quantum efficiency for the photoelectric conversion at $500 \mathrm{~nm}$ for the steam-treated FC-containing electrodes prepared on the steam-treated dye-free layers (steam-treated WE-Tn-FC3) and crystalline titania layers (steam-treated WE-Tcn-FC3) compared to the untreated FC-containing electrodes prepared on the untreated dye-free layers (untreated WE-Tn-FC3). The efficiencies of the former two electrodes were much higher than those of the untreated electrodes. The steam-treated electrode having 3 layers of the dye-free titania, the steam-treated WE-T3-FC3, exhibited a $70 \%$ efficiency compared to the electrode having 3 layers of the heated titania, the steam-treated WE-Tc3-FC3. The 
electrical conductivity of the steam-treated film was close to that of the crystalline titania film, corresponding to their resistivity.

(Fig. 17)

Fig. 18 shows the UV absorption and photocurrent spectra of the untreated titania gel, steam-treated titania gel, and crystalline titania electrodes. The absorption and photocurrent values of the untreated and steam-treated titania gels were much lower than those of the crystalline titania because the steam-treated electrode had a much lower crystallinity and the untreated electrode was amorphous. The low crystallinity indicates a low density of states in the titania, causing the low absorption efficiency and electrical conductivity. However, a relatively high photoinduced electron transport was observed in the steam-treated electrodes as shown in Fig. 17. The light absorption efficiency due to the dye should be much higher than that of the titania. The high photoelectron density was obtained in the conduction band of the dye-containing titania, and the electrons were injected into the other dye-containing titania and the dye-free titania. The dye-containing titania layers functioned as efficient sensitizers. The electrical conductivity of the steam-treated titania was close to that of the crystalline titania prepared by heating, even though the absorption efficiency of the former was lower than that of the latter. We suggest the possible electron transport on the titania particle surface rather than in the bulk in such a low crystalline titania [34]. The adsorbed water molecules enhanced the mobility of the charge 
carriers on the surface. The resistivity value observed for the steam-treated film can indicate the resistivity on the titania particle surface having some ionic species. Therefore, the dye-sensitization can be favorable for the surface carrier production rather than the direct adsorption.

(Fig. 18)

\section{Conclusions}

The spectroscopic and photoelectric measurements of the amorphous dye-containing titania gel and steam-treated nanocrystalline titania films were investigated in order to clarify the photoinduced electron transport in the titania. The influences of the structure and morphology of the multilayered film on the photoelectron transport and electrically conductive properties were discussed. The photocurrent was observed only from the layer contacting the crystalline titania foundation prepared on the ITO electrode. The electron transport from the upper layers was difficult in the amorphous titania film. However, the upper layers apparently increased the photocurrent because they functioned as blocking layers for the back electron transfer to the electrolyte. The steam treatment of the electrodes improved the electron transport due to crystallization of the amorphous titania accompanied by enhancement of its electrical conductivity. The UV photocurrent values of the steam-treated titania were much lower than that of the crystalline titania due to its 
significantly lower crystallinity. However, the efficiency of the dye-sensitized electron transport in the steam-treated titania film was $70 \%$ of that in the anatase film prepared by heating at $773 \mathrm{~K}$. The light absorption efficiency due to the dye should be much higher than that of the titania. The high photoelectron density was obtained in the conduction band of the dye-containing titania, and the electrons were injected into the dye-free titania. The dye-containing titania layers functioned as efficient sensitizers.

\section{Acknowledgements}

This work was supported by the Ministry of Education, Science, Sports and Culture, Grant-in-Aid for Young Scientists (B), 2008-2009.

\section{References}

[1] B. O'Regan, M. Grätzel, Nature 353 (1991) 737-740.

[2] M. K. Nazeeruddin, A. Kay, I. Rodicio, R. Hamphry-Baker, E. Müeller, P. Liska, N. Vlachopoulos, M. Grätzel, J. Am. Chem. Soc. 115 (1993) 6382-6390.

[3] M. Grätzel, J. Photochem. Photobiol. C: Photochem. Rev. 4 (2003) 145-153.

[4] K. Murakoshi, G. Kano, Y. Wada, S. Yanagida, H. Miyazaki, M. Matsumoto, S. Murasawa, J. Electroanal. Chem. 396 (1995) 27-34. 
[5] K. Kalyanasundaram, M. Grätzel, Coordination Chem. Rev. 177 (1998) 347-414.

[6] J. He, F. Chen, J. Zhao, H. Hidaka, Colloids Surfaces A: Physicochem. Eng. Aspects 142 (1998) 49-57.

[7] C. Wang, C. Liu, Y. Wang, T. Shen, J. Colloid Interface Sci. 197 (1998) 126-132.

[8] M. Hilgendorff, V. Sundström, J. Phys. Chem. B 102 (1998) 10505-10514.

[9] G. Ramakrishna, H. N. Ghosh, J. Phys. Chem. B 105 (2001) 7000-7008.

[10] G. Benkö, M. Hilgendorff, A. P. Yartsev, V. Sundström, J. Phys. Chem. B 105 (2001) 967-974.

[11] G. Benkö, B. Skårman, R. Wallenberg, A. Hagfeldt, V. Sundström, A. P. Yartsev, J. Phys. Chem. B 107 (2003) 1370-1375.

[12] S. Pelet, M. Grätzel, J. E. Moser, J. Phys. Chem. B 107 (2003) 3215-3224.

[13] G. Ramakrishna, A. Das, H. N. Ghosh, Langmuir 20 (2004) 1430-1435.

[14] D. El Mekkawi, M. S. A. Abdel-Mottaleb, Int. J. Photoenergy 7 (2005) 95-101.

[15] G. D. Sharma, P. Balraju, M. Kumar, M. S. Roy, Mater. Sci. Eng. B 162 (2009) 32-39.

[16] H. Nishikiori, N. Tanaka, T. Kitsui, T. Fujii, J. Photochem. Photobiol. A: Chem. 179 (2006) 125-129.

[17] T. Kitsui, H. Nishikiori, N. Tanaka, T. Fujii, J. Photochem. Photobiol. A: Chem. 192 (2007) 220-225.

[18] H. Nishikiori, W. Qian, M. A. El-Sayed, N. Tanaka, T. Fujii, J. Phys. Chem. C 111 
(2007) 9008-9011.

[19] H. Nishikiori, Y. Uesugi, N. Tanaka, T. Fujii, J. Photochem. Photobiol. A: Chem. 207 (2009) 204-208.

[20] H. Nishikiori, Y. Uesugi, S. Takami, R. A. Setiawan, T. Fujii, W. Qian, M. A. El-Sayed, J. Phys. Chem. C 115 (2011) 2880-2887.

[21] K. Yanagisawa, J. Ovenstone, J. Phys. Chem. B 103 (1999) 7781-7787.

[22] T. Fujii, A. Ishii, N. Takusagawa, M. Anpo, Res. Chem. Intermed. 17 (1992) 1-14.

[23] N. O. Mchedlov-Petrossyan, V. N. Kleshchevnikova, J. Chem. Soc. Faraday Trans. 90 (1994) 629-640.

[24] P. Tarte, Physics of Non-Crystalline Solids, North Holland, 1965, 549-565.

[25] M. L. Galzada, L. Delolmo, J. Non-Cryst. Solids 121 (1990) 413-416.

[26] S. Ben Amor, G. Baud, J. P. Besse, M. Jacquet, Mater. Sci. Eng. B 47 (1997) 110-118.

[27] L. Castañeda, J. C. Alonso, A. Ortiz, E. Andrade, J. M. Saniger, J. G. Bañuelos, Mater. Chem. Phys. 77 (2002) 938-944.

[28] G. Gusmano, G. Montesperelli, P. Nunziante, E. Traversa, A. Montenero, M. Braghini, G. Mattogno, A. Bearzotti, J. Ceram. Soc. Jpn. 101 (1993) 1095-1100.

[29] M. Ivanda, S. Musić, S. Popović, M. Gotić, J. Mol. Struct. 480481 (1999) 645-649.

[30] J. Trimboli, M. Mottern, H. Verweij, P. K. Dutta, J Phys. Chem. B 110 (2006) $5647-5654$ 
[31] J. Yu, X. Zhao, Q Zhao, Mater. Chem. Phys. 69 (2001) 25-29.

[32] H. Nishikiori, T. Sato, S. Kubota, N. Tanaka, Y. Shimizu, T. Fujii, Res. Chem. Intermed. 38 (2012) 595-613.

[33] K. Kajihara, T. Yao, Phys. Chem. Chem. Phys. 1 (1999) 1979-1983.

[34] D. Eder, R. Kramer, Phys. Chem. Chem. Phys. 5 (2003) 1314-1319. 


\section{Scheme title and figure captions}

Scheme 1 Structures of the working electrodes.

Figure 1 Visible (a) absorption and (b) photocurrent spectra of the untreated FC-titania electrodes, WE-FCn $(\mathrm{n}=1,2,3,5$, and 7).

Figure $2 I-V$ curves of the untreated FC-titania electrodes, WE-FCn $(\mathrm{n}=1,2,3,5$, and 7$)$.

Figure 3 Visible (a) absorption and (b) photocurrent spectra of the untreated EY-titania electrodes, WE-EYn $(\mathrm{n}=1,2,3,5$, and 7).

Figure $4 \quad I-V$ curves of the untreated EY-titania electrodes, WE-EYn $(\mathrm{n}=1,2,3,5$, and 7$)$.

Figure 5 Visible (a) absorption and (b) photocurrent spectra of the untreated FC-titania electrodes, WE-FC1 and WE-FC1-Tn $(\mathrm{n}=1,2,4$, and 6).

Figure $6 \quad I-V$ curves of the untreated FC-titania electrodes, WE-FC1 and WE-FC1-Tn $(\mathrm{n}=$ $1,2,4$, and 6).

Figure 7 Visible (a) absorption and (b) photocurrent spectra of the untreated EY-titania electrodes, WE-EY1 and WE-EY1-Tn $(\mathrm{n}=1,2,4$, and 6).

Figure $8 \quad I-V$ curves of the untreated EY-titania electrodes, WE-EY1 and WE-EY1-Tn $(\mathrm{n}=$ $1,2,4$, and 6). 
Figure 9 Photocurrent spectra of (a) the untreated FC-titania electrodes, WE-FC3 and WE-Tn-FC3, and (b) the untreated EY-titania electrodes, WE-EY3 and WE-Tn-EY3 ( $\mathrm{n}=1,2$, 3 , and 4).

Figure 10 SEM images of (a) the steam-treated titania and (b) the crystalline titania.

Figure 11 XRD patterns of the untreated and steam-treated titania gel and the crystalline titania.

Figure 12 FTIR spectra of the untreated and steam-treated titania gel and the crystalline titania.

Figure 13 XPS spectra of (a) Ti 2p and (b) O 1s for (1) the untreated and (2) steam-treated titania and (3) the crystalline titania.

Figure 14 Visible (a) absorption and (b) photocurrent spectra of the untreated dye-titania electrodes, WE-FC3, WE-EY3, WE-FC3-EY3, and WE-EY3-FC3.

Figure 15 Visible (a) absorption and (b) photocurrent spectra of the steam-treated dye-titania electrodes, WE-FC3, WE-EY3, WE-FC3-EY3, and WE-EY3-FC3.

Figure 16 Photocurrent spectra of the steam-treated FC-titania electrodes, the steam-treated WE-FC3 and WE-Tn-FC3 ( $\mathrm{n}=3,5$, and 7).

Figure 17 Quantum efficiency for the photoelectric conversion at $500 \mathrm{~nm}$ of the steam-treated FC-titania electrodes prepared on the steam-treated dye-free titania layers (steam-treated WE-Tn-FC3) and crystalline titania layers (steam-treated WE-Tcn-FC3) 
compared to that of the untreated FC-titania electrodes prepared on the untreated dye-free titania layers (untreated WE-Tn-FC3).

Figure 18 UV (a) absorption and (b) photocurrent spectra of the untreated titania gel, steam-treated titania gel, and crystalline titania electrodes.

Supporting information

Figure S1 Photocurrent density values of the electrodes, the untreated and steam-treated WE-FC3, obtained as a function of light irradiation time. The photocurrent density for each electrode was normalized at each initial value. 
Scheme 1

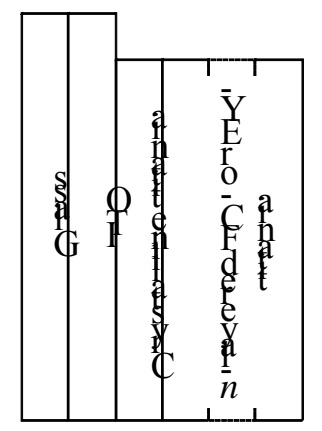

WE-FCn or WE-EYn $(n=1-7)$

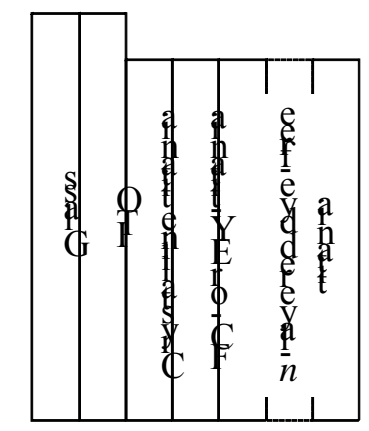

WE-FC1-T $n$ or WE-EY1-Tn $(n=1-6)$

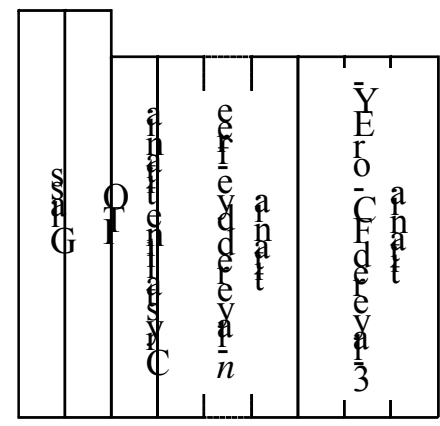

WE-Tn-FC3 or WE-Tn-EY3 $(n=1-7)$

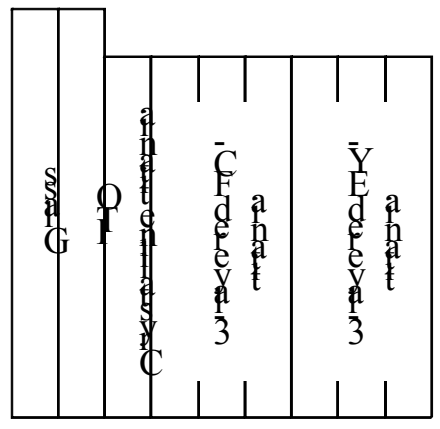

WE-FC3-EY3

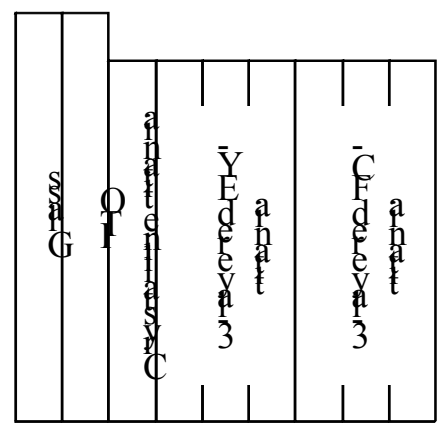

WE-EY3-FC3 
Figure 1
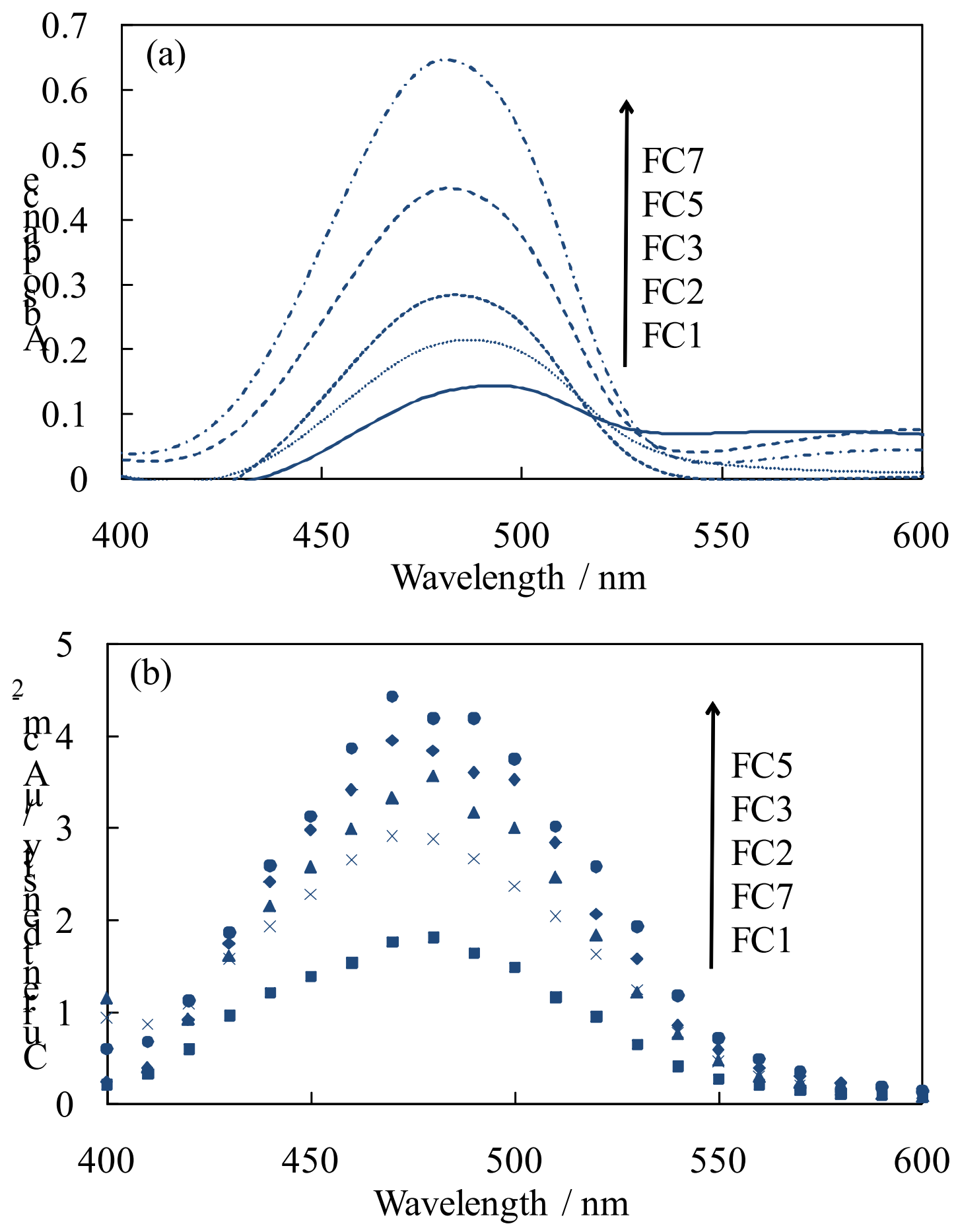
Figure 2

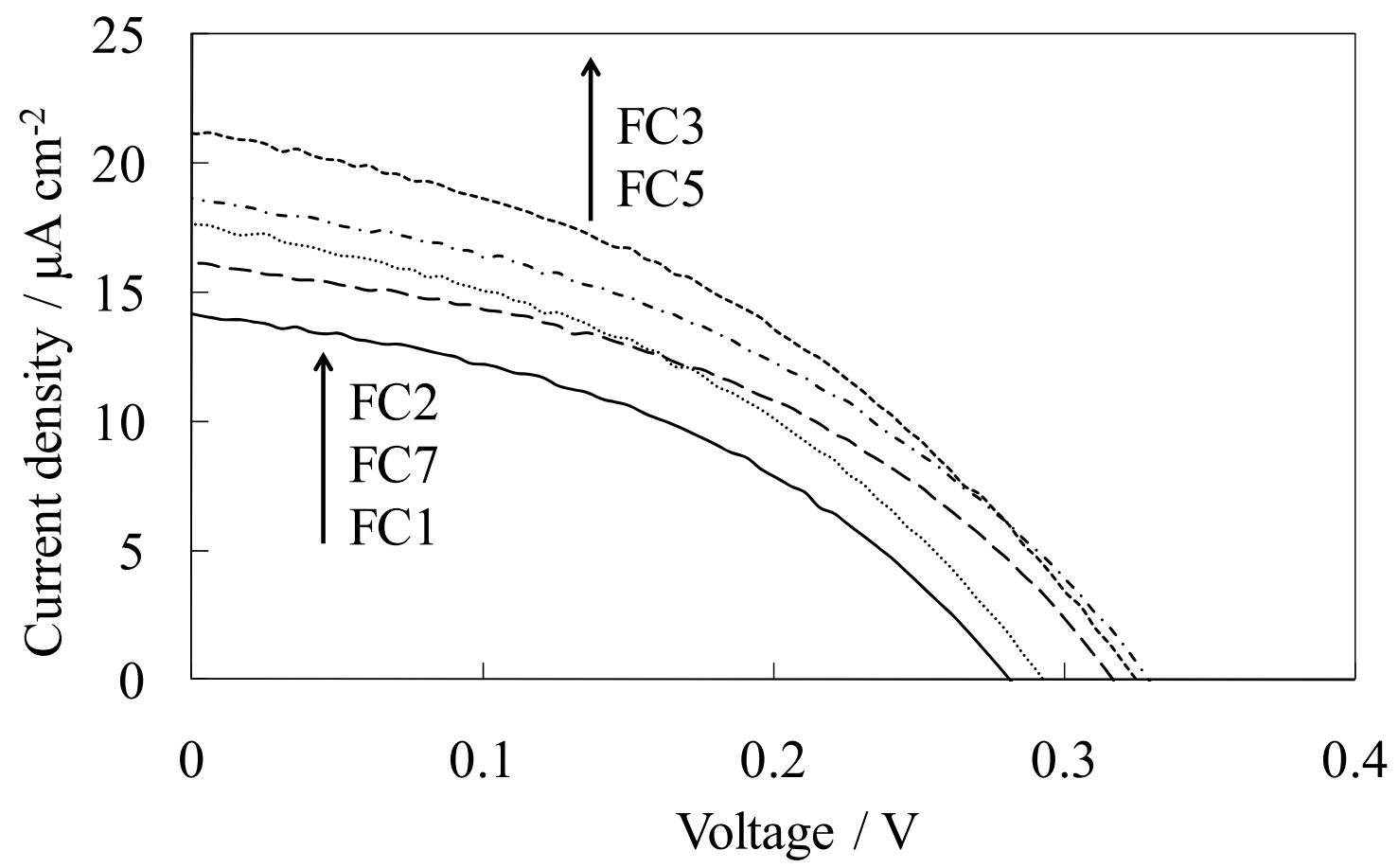


Figure 3
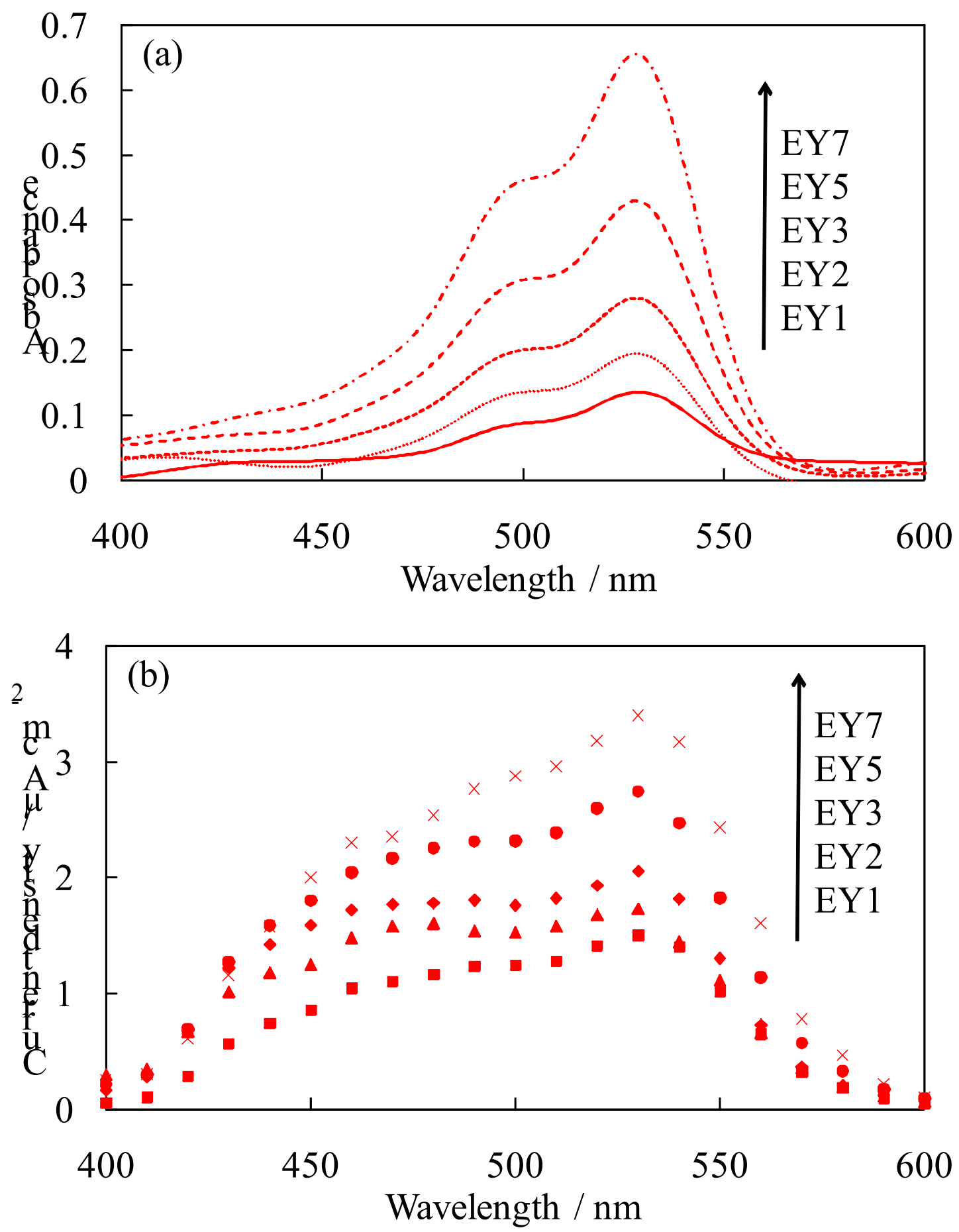
Figure 4

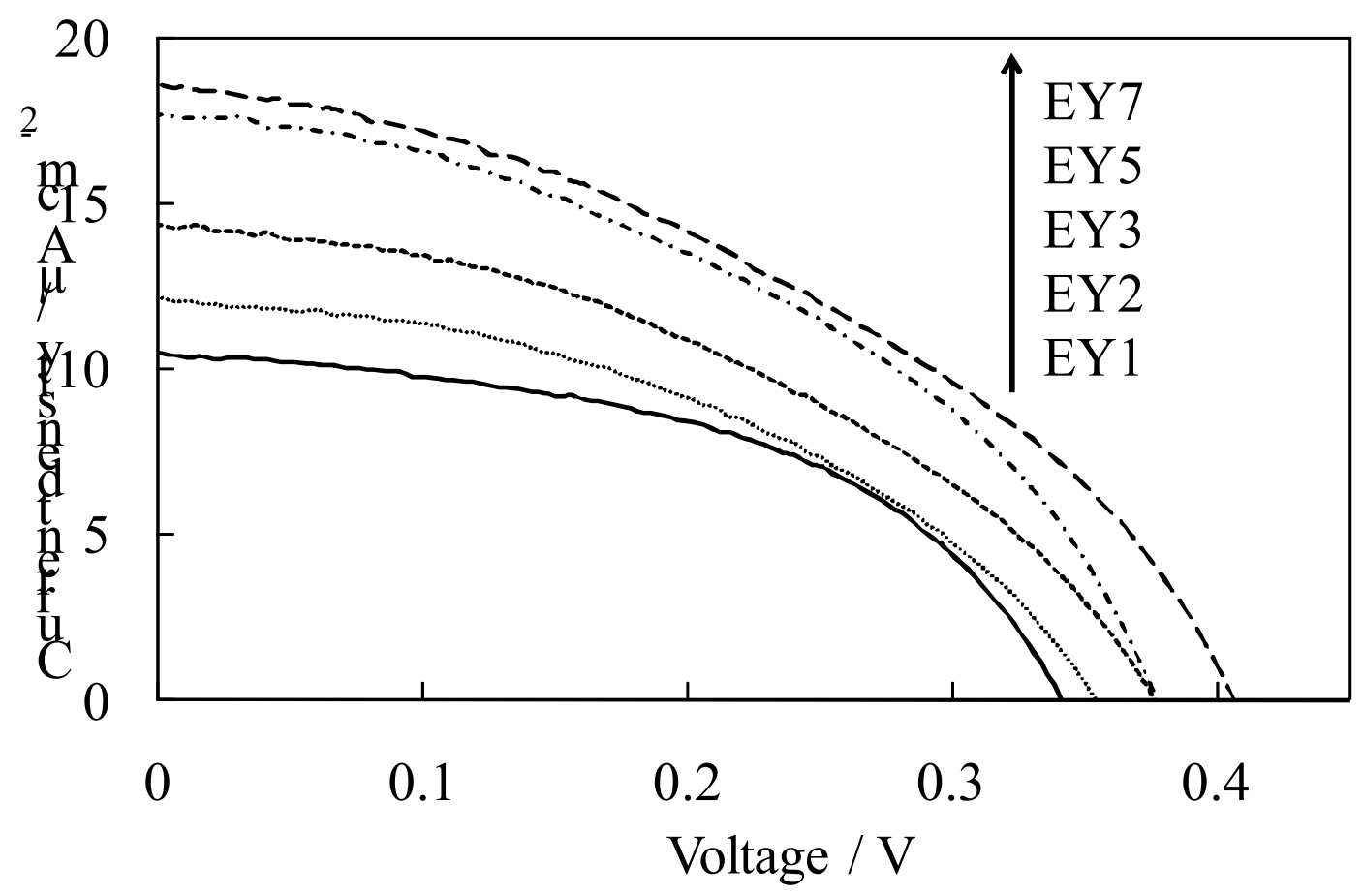


Figure 5
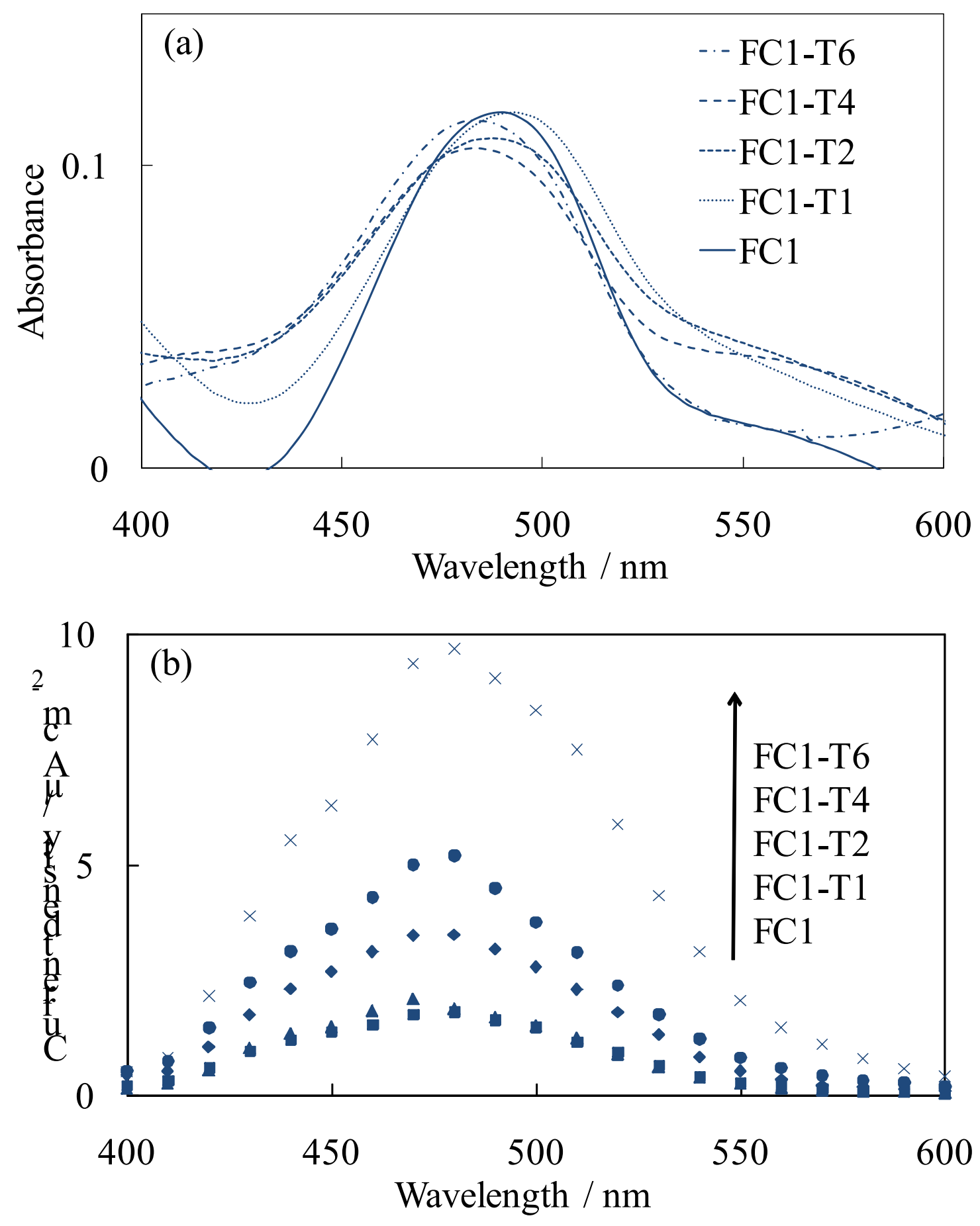
Figure 6

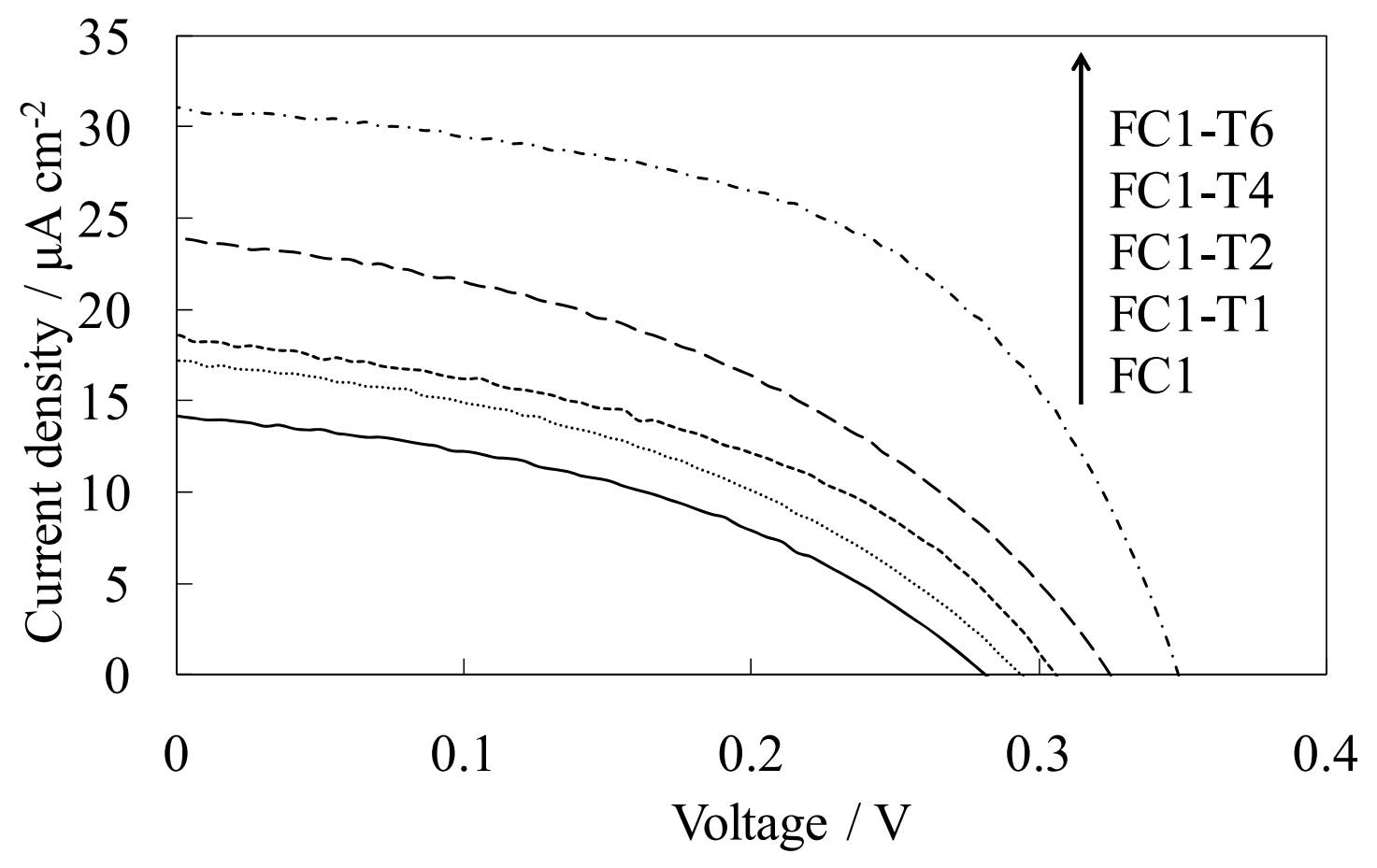


Figure 7
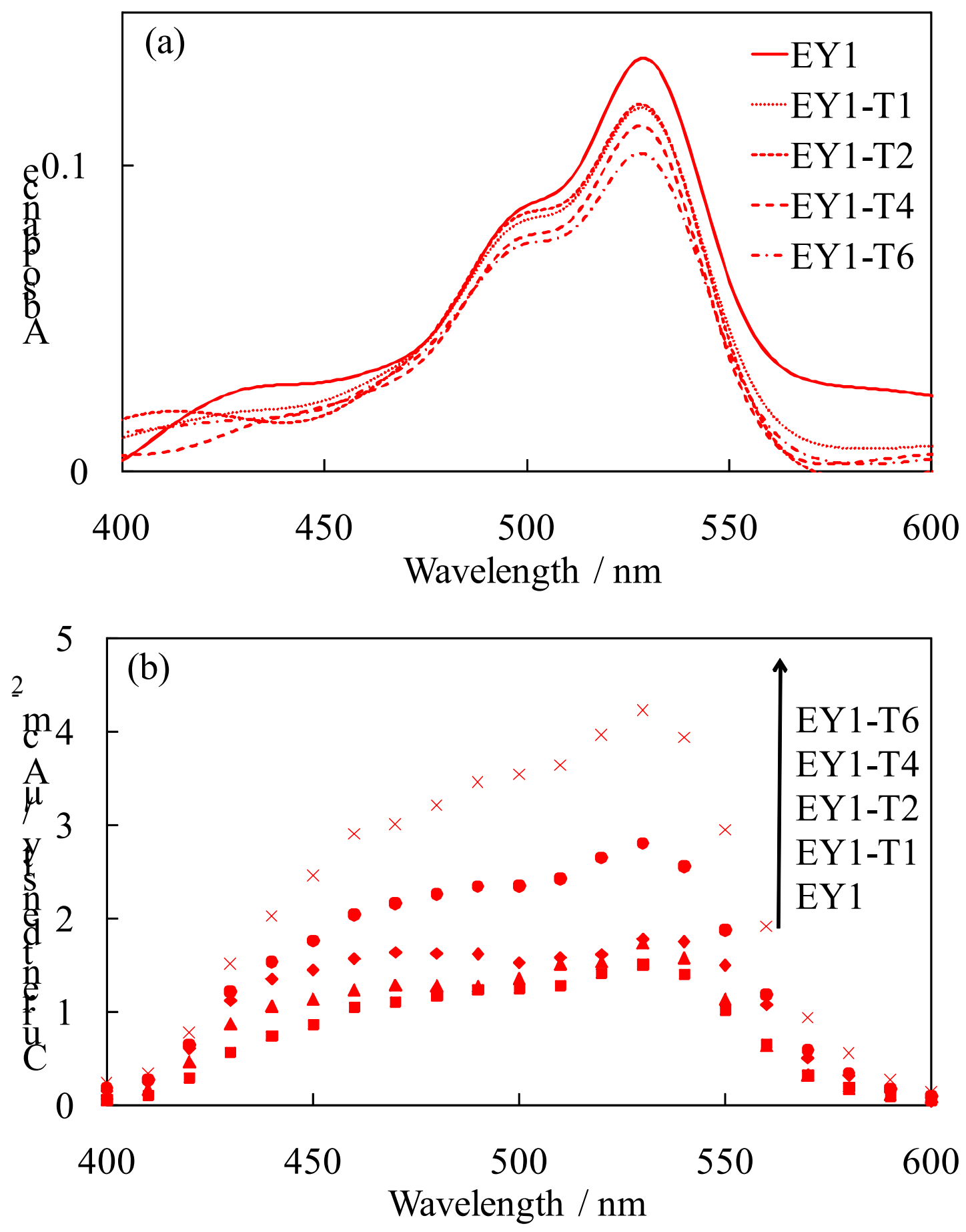
Figure 8

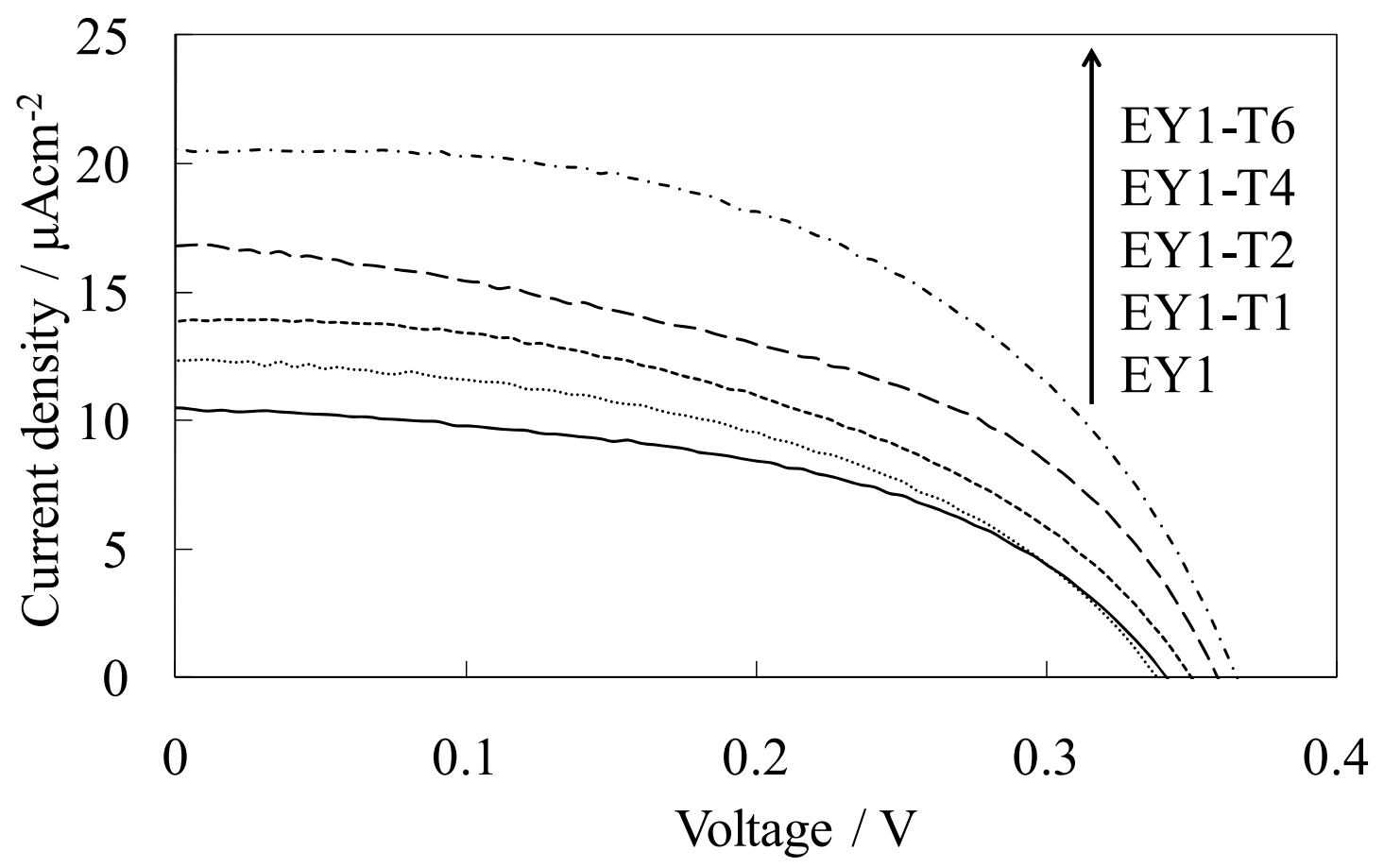


Figure 9
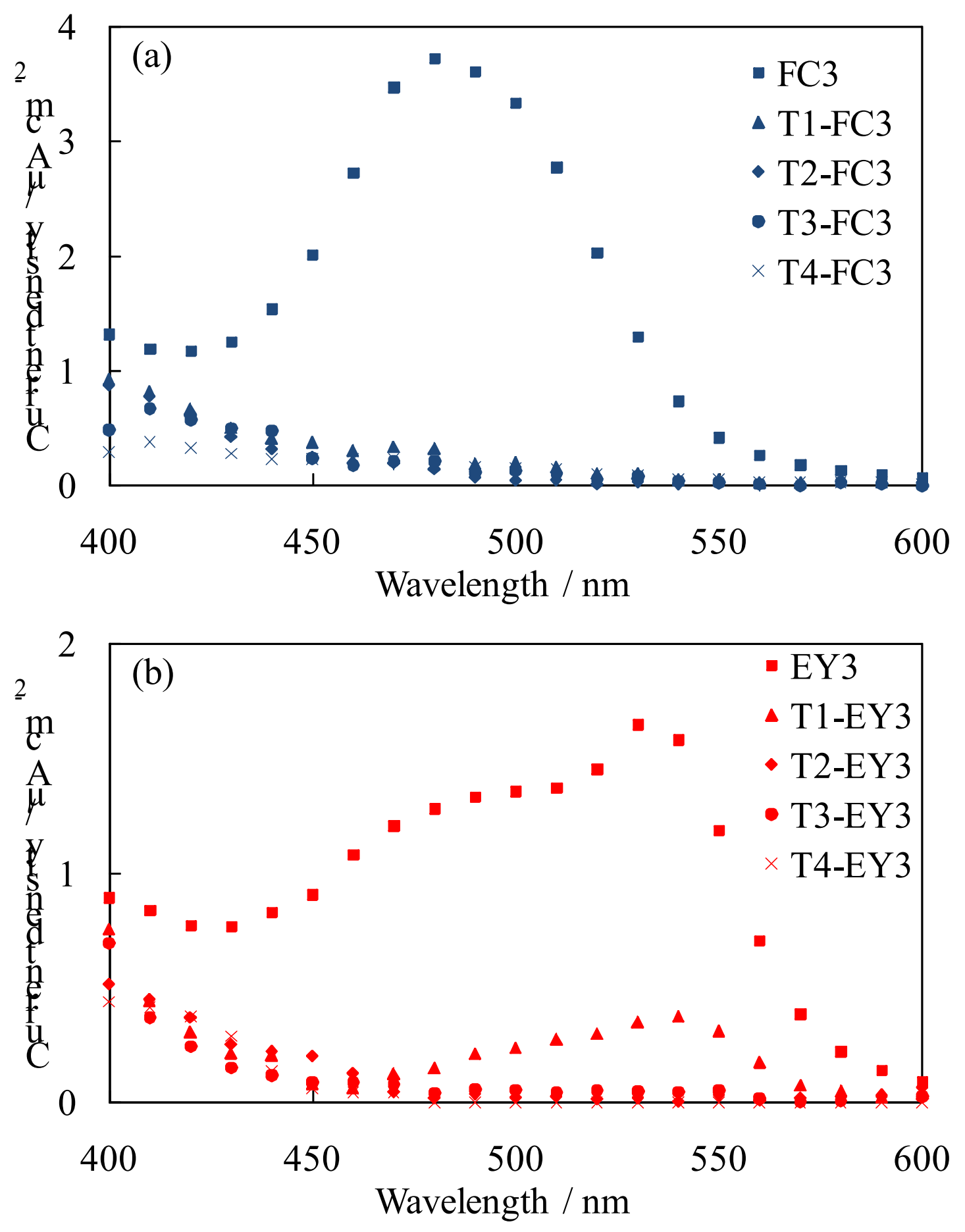
Figure 10
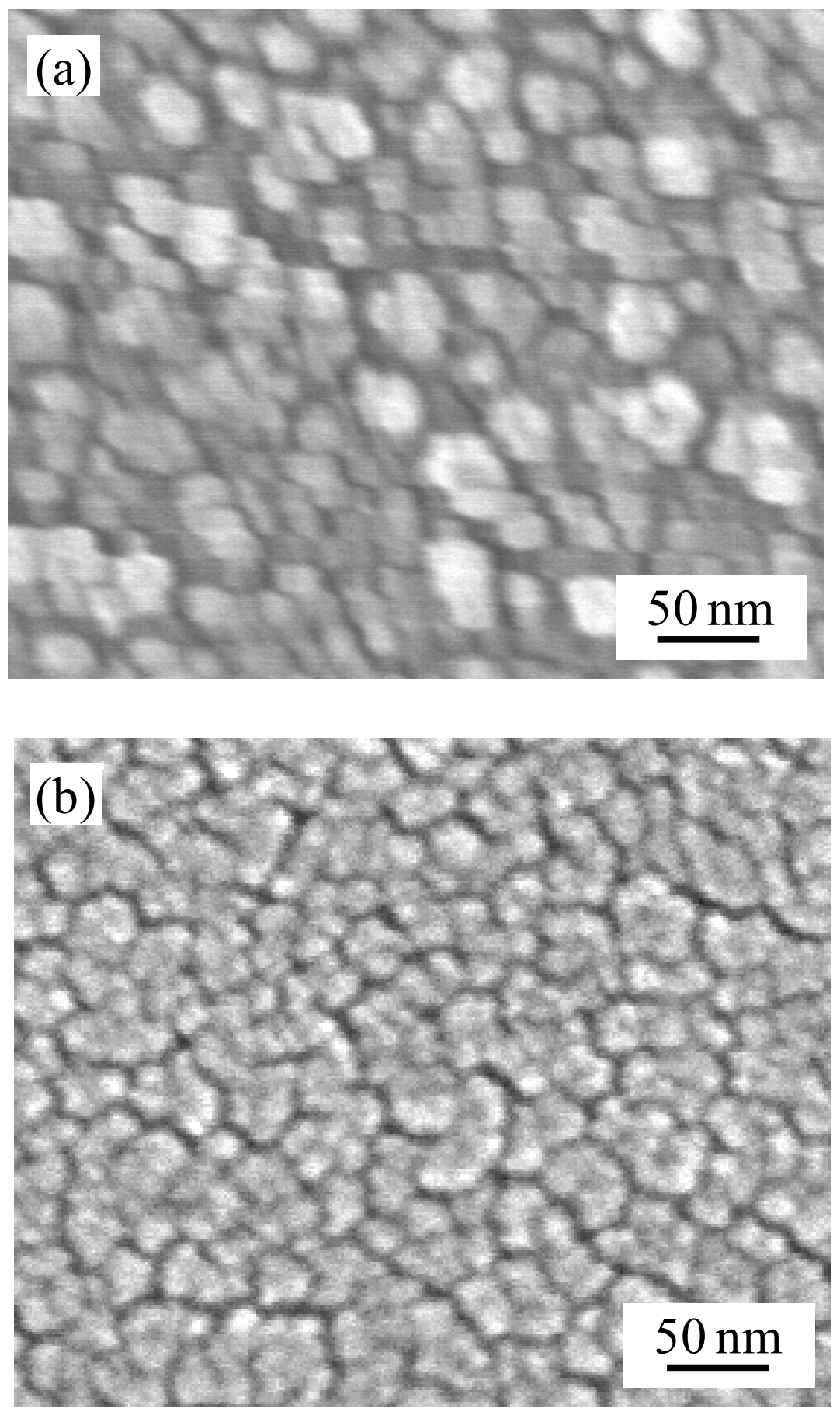
Figure 11

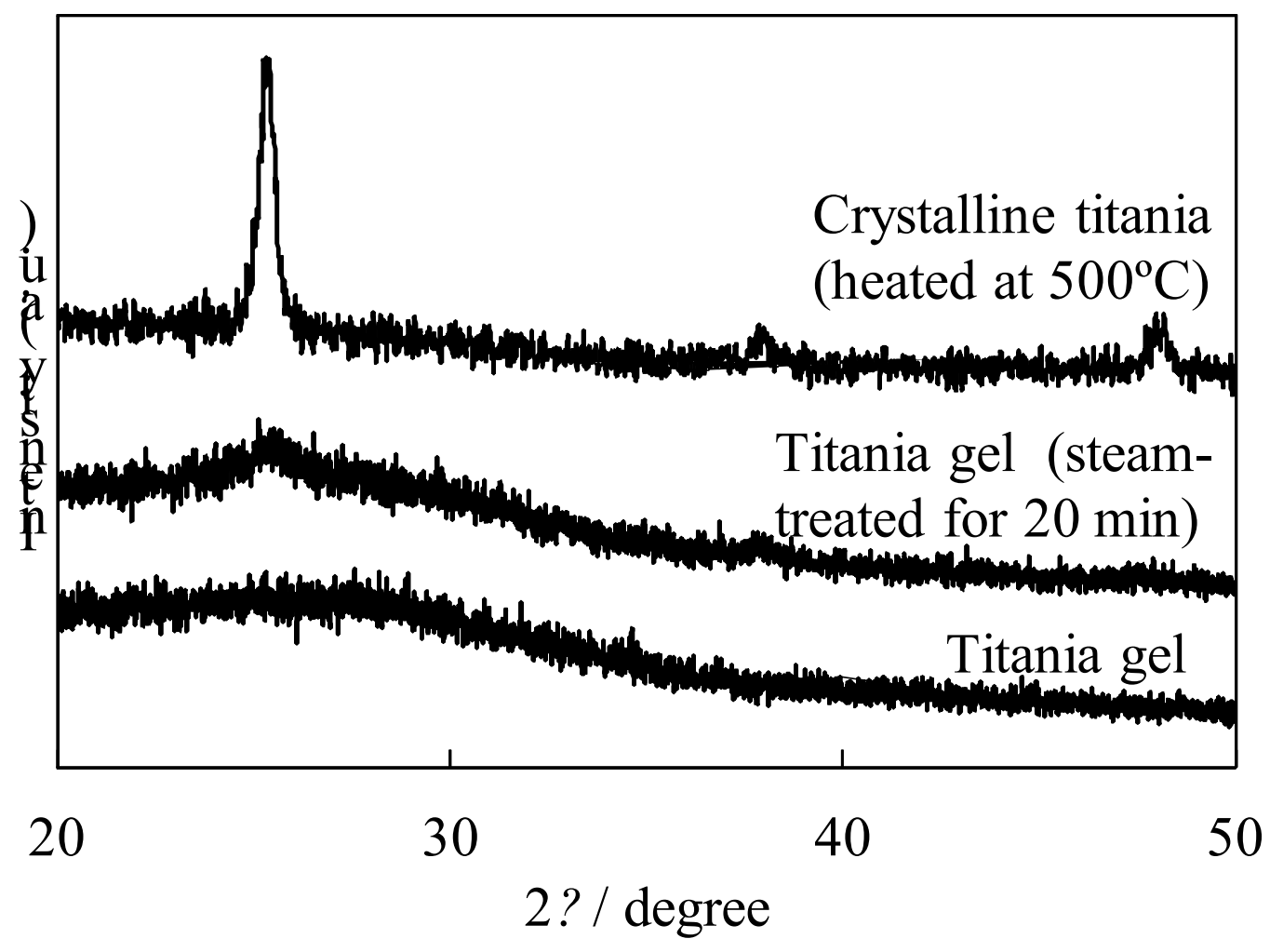


Figure 12

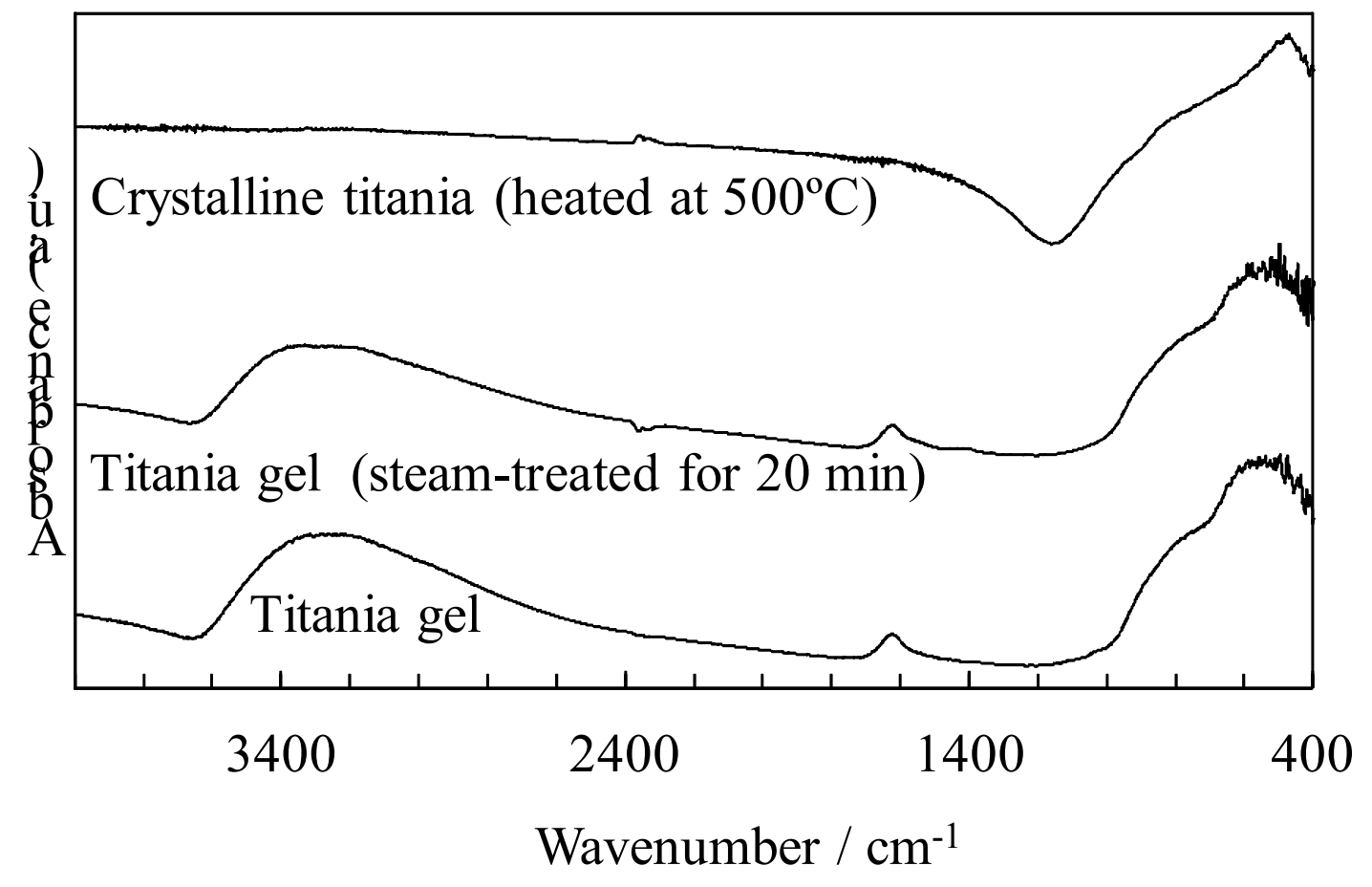


Figure 13
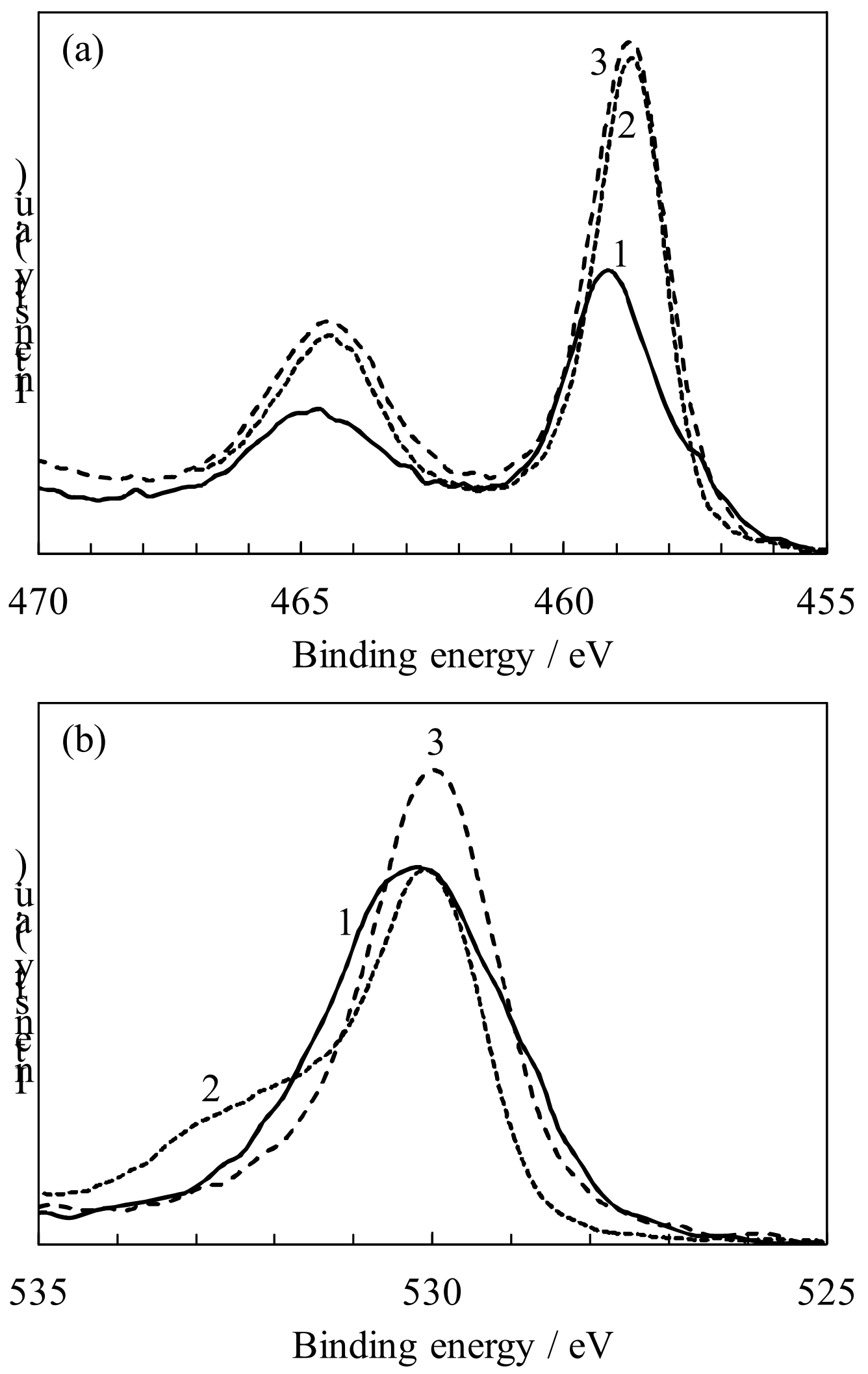
Figure 14
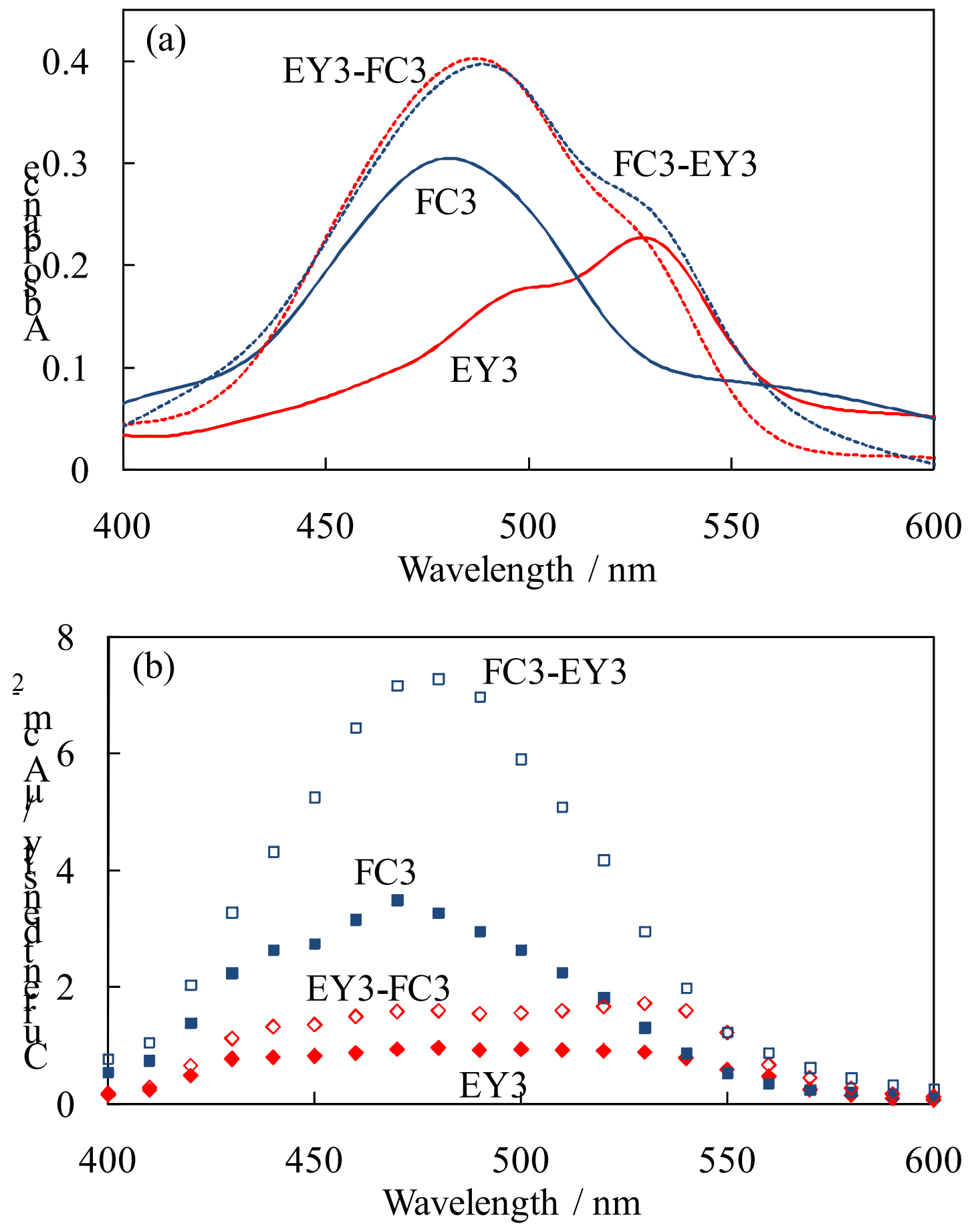
Figure 15
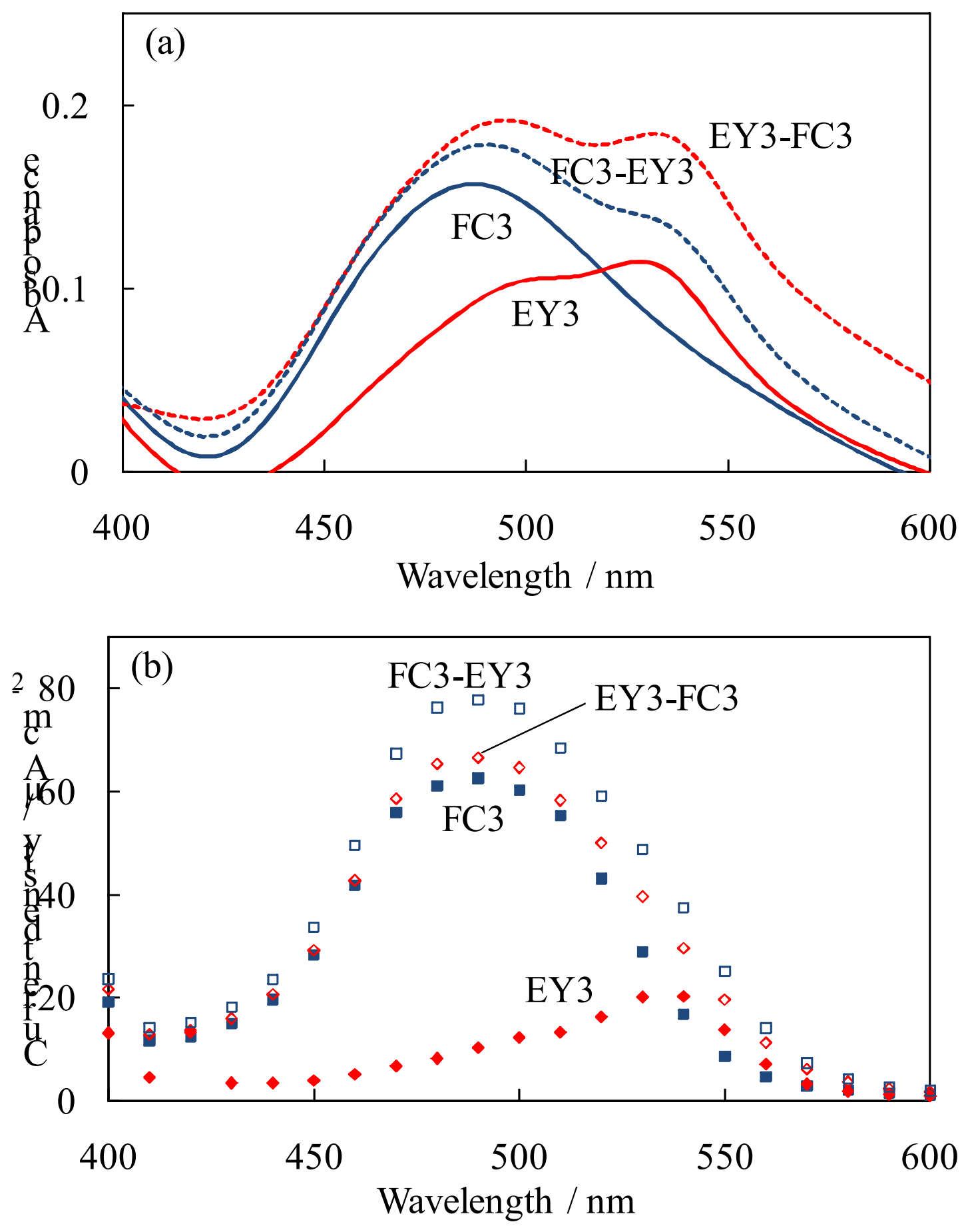
Figure 16

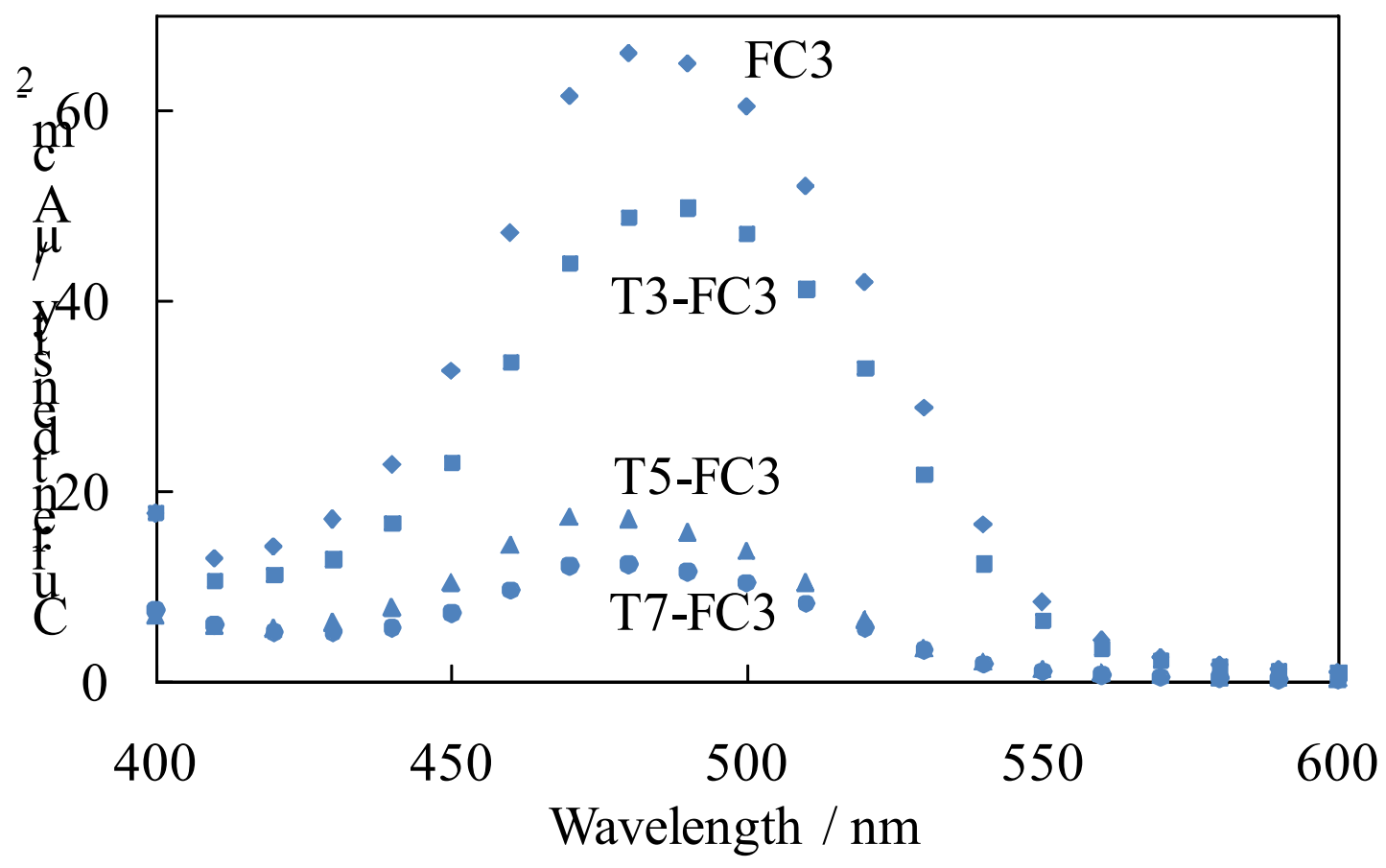


Figure 17

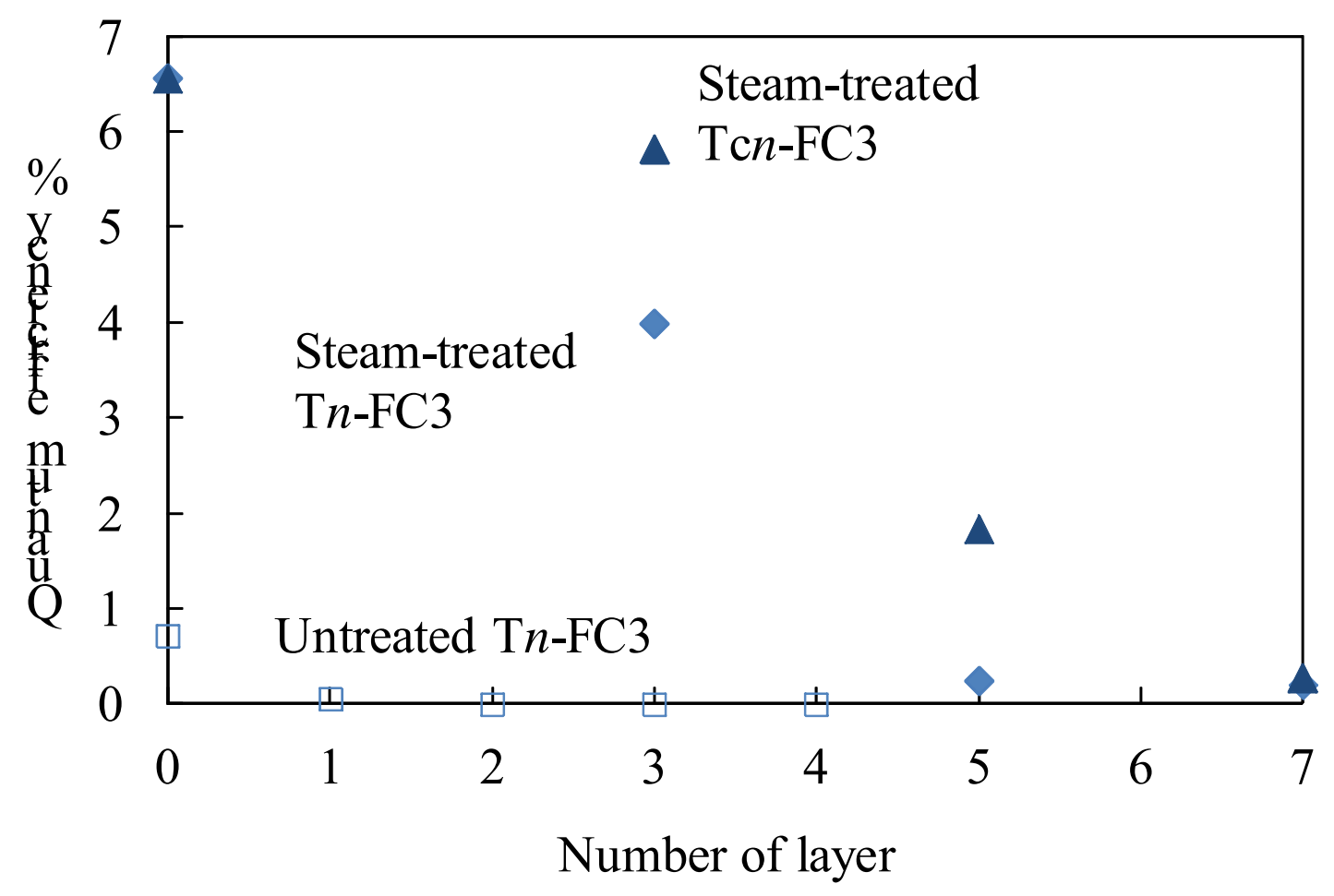


Figure 18
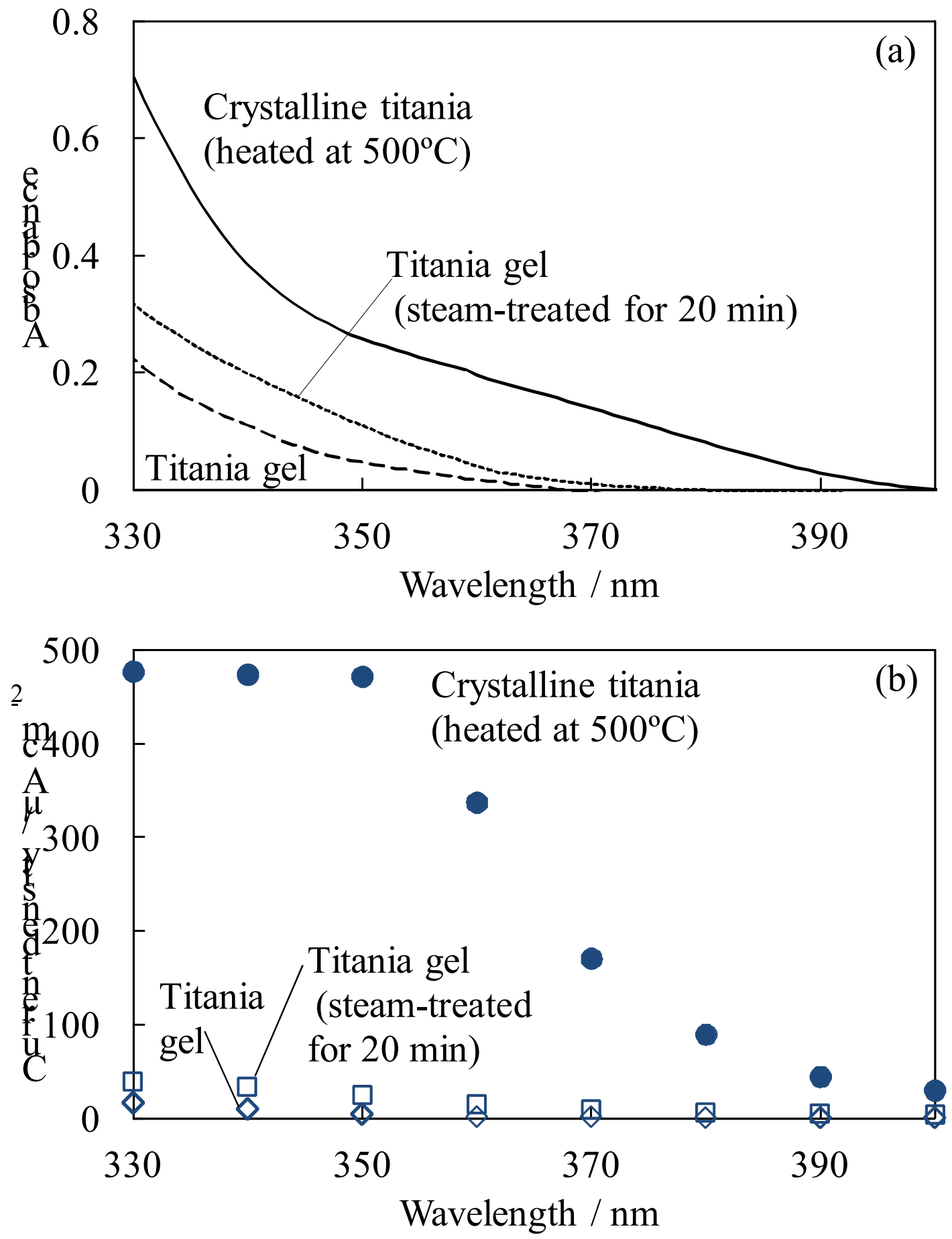
Figure S1

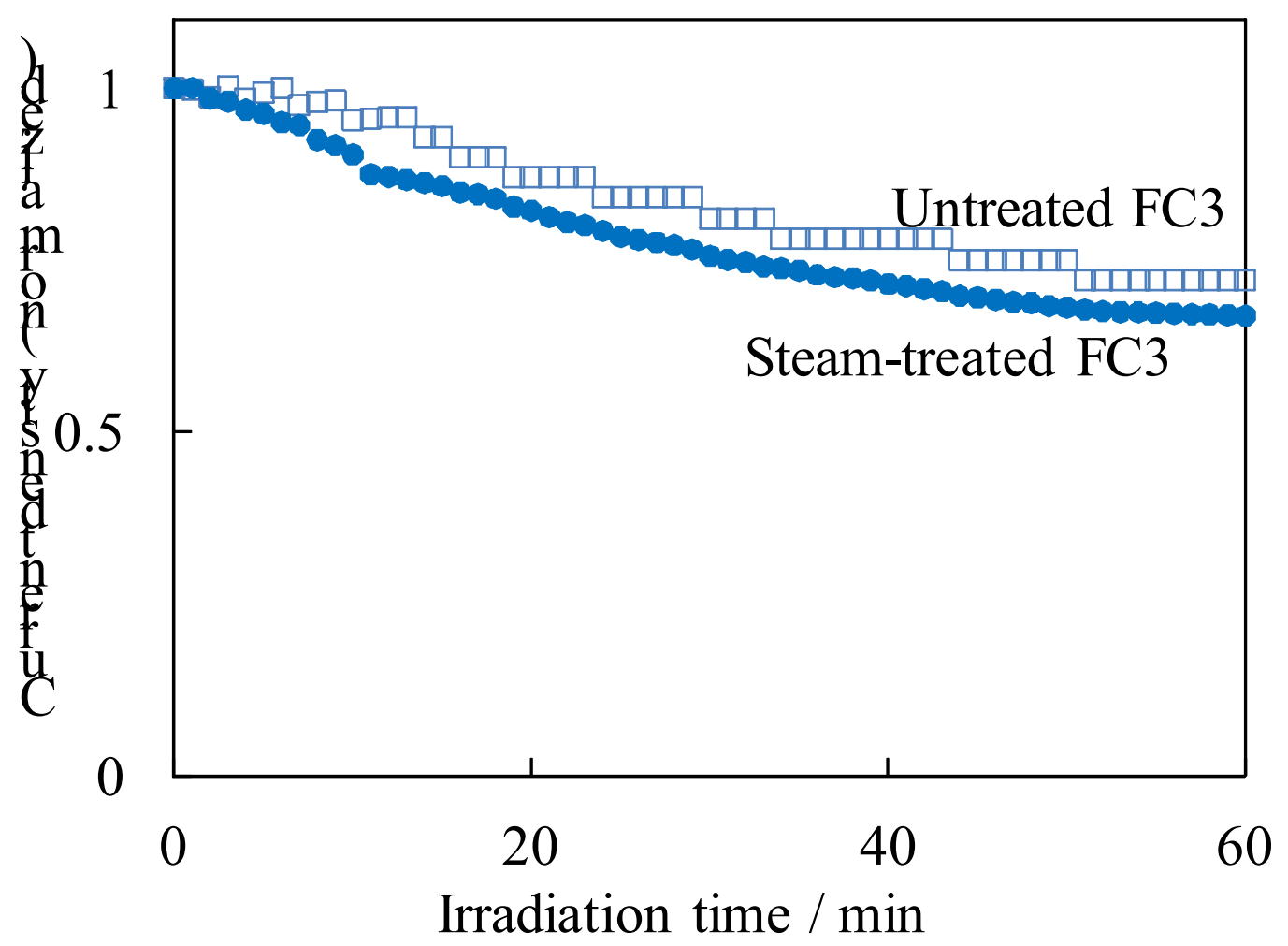

